http://dx.doi.org/10.1016/j.plipres.2013.07.003

\title{
Nonlinear vibrational microscopy applied to lipid biology
}

\author{
Andreas Zumbusch ${ }^{\mathrm{a}}$, Wolfgang Langbein ${ }^{\mathrm{b}}$, Paola Borri ${ }^{\mathrm{b}, \mathrm{c}, *}$ \\ a Department of Chemistry, University of Konstanz, 78457 Konstanz, Germany \\ ${ }^{\mathrm{b}}$ Cardiff University School of Physics and Astronomy, The Parade, Cardiff CF24 3AA, United Kingdom \\ ${ }^{\mathrm{c}}$ Cardiff University School of Biosciences, Museum Avenue, Cardiff CF10 3AX, United Kingdom
}

Keywords:

Multiphoton microscopy

Nonlinear optics

Coherent anti-Stokes Raman scattering

Lipids

\begin{abstract}
Optical microscopy is an indispensable tool that is driving progress in cell biology. It still is the only prac tical means of obtaining spatial and temporal resolution within living cells and tissues. Most promi nently, fluorescence microscopy based on dye labeling or protein fusions with fluorescent tags is a highly sensitive and specific method of visualizing biomolecules within sub cellular structures. It is how ever severely limited by labeling artifacts, photo bleaching and cytotoxicity of the labels. Coherent Raman Scattering (CRS) has emerged in the last decade as a new multiphoton microscopy technique sui ted for imaging unlabeled living cells in real time with high three dimensional spatial resolution and chemical specificity. This technique has proven to be particularly successful in imaging unstained lipids from artificial membrane model systems, to living cells and tissues to whole organisms. In this article, we will review the experimental implementations of CRS microscopy and their application to imaging lipids. We will cover the theoretical background of linear and non linear vibrational micro spectroscopy neces sary for the understanding of CRS microscopy. The different experimental implementations of CRS will be compared in terms of sensitivity limits and excitation and detection methods. Finally, we will provide an overview of the applications of CRS microscopy to lipid biology.
\end{abstract}

\section{Contents}

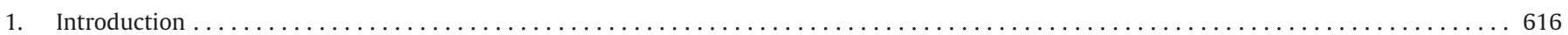

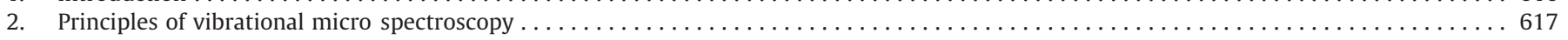

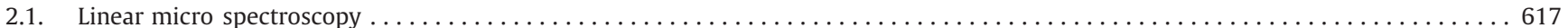

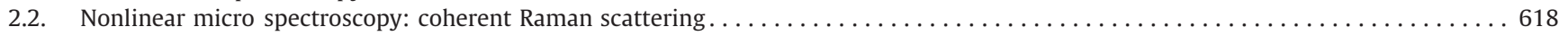

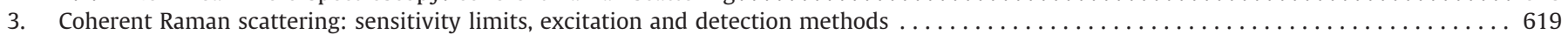

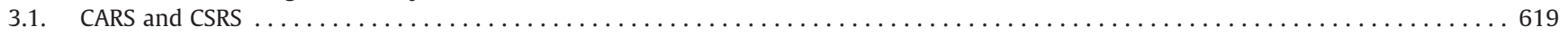

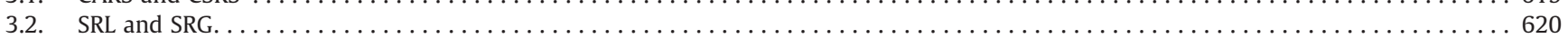

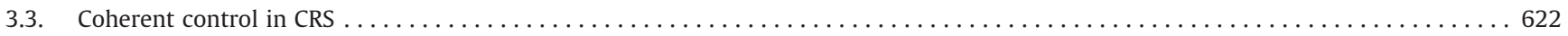

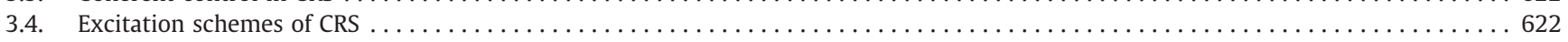

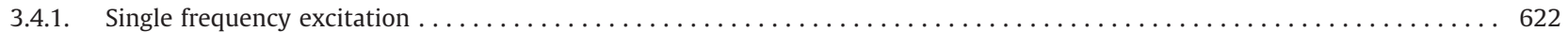

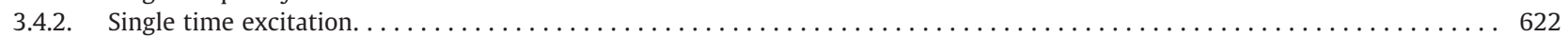

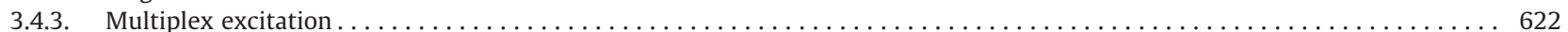

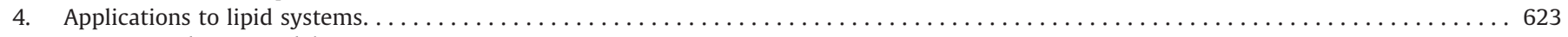

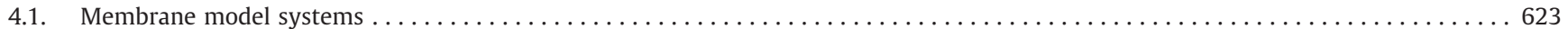

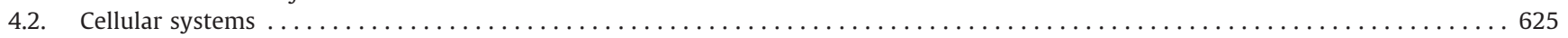

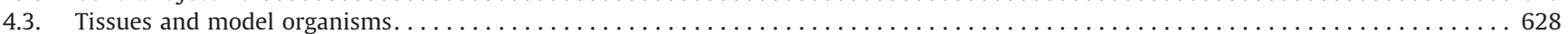

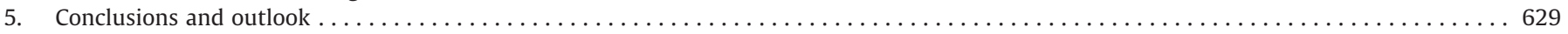

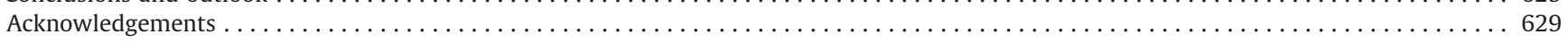

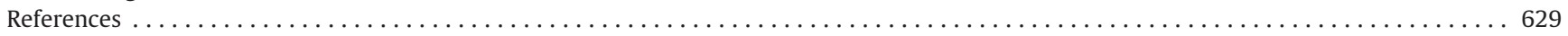

* Corresponding author at: Cardiff University School of Physics and Astronomy, The Parade, Cardiff CF24 3AA, United Kingdom. Tel.: +44 029 208 79356 ; fax: +44 029208 74305.

E-mail addresses: borrip@cardiff.ac.uk, borrip@cf.ac.uk (P. Borri). 


\section{Introduction}

For centuries, developments in optical microscopy have led to new insights in cell biology which in turn stimulated the evolution of these optical tools. In particular, the advent of the laser provid ing a bright light source with a well defined spatial and temporal mode has had a profound impact on the contrast techniques avail able in optical microscopy. Today, most prominent among the la ser based microscopy techniques are confocal and two photon fluorescence (TPF) microscopy [1], which together with the devel opment of highly specific fluorescent labeling techniques have shaped modern cell biology. In confocal microscopy, continuous wave lasers are employed to excite the fluorophore of interest via one photon absorption. An image is generated by raster scan ning the focused beam across the sample and the emitted fluores cence is recorded sequentially with a single channel detector. Three dimensional (3D) spatial resolution is achieved by filtering the detected light by a confocal pinhole, suppressing out of focus light. An alternative way to obtain 3D resolution is realized in TPF microscopy where two near infrared photons, for which one photon absorption is negligible, are absorbed simultaneously by the fluorophore. As in confocal fluorescence, an image is obtained by raster scanning the focused beam over the sample while collect ing the emitted fluorescence into a single channel detector. Since two photon absorption is non linear and occurs dominantly in the focal volume where high photon densities are reached, TPF has an intrinsic 3D resolution without the need of a detection pin hole. In turn the collection efficiency is improved, since light gen erated in the focal volume and subsequently scattered within the sample is not rejected. An important advantage of TPF is the use of near infrared light which enables deeper penetration into thick specimens due to the reduced Rayleigh scattering at longer wave lengths and the reduced one photon absorption from endogenous cellular material. Importantly, TPF requires pulsed lasers to gener ate sufficient photon densities at the pulse peak while maintaining moderate average powers. Typical laser sources for TPF micros copy, which are now found in many bio medical laboratories, pro vide pulses with durations of approximately $100 \mathrm{fs}$ and repetition rates on the order of $100 \mathrm{MHz}$.

The success of TPF microscopy paved the way for research into the use of other non linear optical phenomena for the generation of contrast in high resolution optical microscopy. It was quickly realized that non linear processes are especially well suited for the investigation of unlabeled samples. While fluorescence label ing is a mature technique to specifically tag and hence visualize biomolecules in living cells, it also has inherent drawbacks. Firstly, fluorescence labeling requires a sample preparation procedure, conditioning the types of samples which can be studied, and add ing time delays and cost. Secondly, the labeling changes the system under investigation. Although in many cases these changes are acceptable, there are numerous examples of labeling artifacts. In the field of lipid biology, this concerns for example the observation of fusion of lipid droplets which can be induced by standard label ing protocols [2 4]. A third problem of fluorescence microscopy is the photobleaching of organic fluorophores. Electronic excitation of organic fluorophores leads with a certain probability to chemical reactions towards products which do not absorb at the excitation wavelength and/or do not fluoresce. This often severely limits the observation times in fluorescence microscopy and is accompanied by cytotoxic effects.

One should note that many linear label free optical microscopy techniques are widely used already for decades. Examples are dif ferential interference contrast (DIC) microscopy and phase contrast microscopy. The contrast in these techniques relies on refractive index differences in the sample which gives little information on the chemical composition. On the other hand, the ability to distin guish chemically specific structures in cells and tissues (e.g. lipid vesicles from other cellular vesicles) is crucial to the understanding of their functional behavior within the cellular machinery. One way to generate chemical contrast without the need for external labeling is to exploit the vibrational spectra of biomolecules. Vibra tional resonances depend on the masses of the constituting atoms and their respective bond strengths. A typical vibrational spectrum therefore contains a large number of resonances relating to the set of vibrational modes of the molecule reflecting the chemical com position of the sample. Conventional techniques for vibrational $\mathrm{mi}$ cro spectroscopy are the absorption of infrared (IR) light and Raman scattering (see Section 2.1). IR absorption microscopy is a well established technique. Yet, it is much more widely used in material science than in biological applications. The main two rea sons for this are the poor spatial resolution achievable with IR wavelength and the strong IR absorption of water, which makes IR investigations of biological samples difficult. Raman microscopy, by contrast, utilizes light sources in the visible wavelength range. For this reason, it delivers spatial resolutions comparable to those achievable in confocal fluorescence microscopy. The application of Raman microscopy is not hampered by the presence of water in the sample. However, Raman scattering is weak, such that it can be overwhelmed even by weak autofluorescence in the sample. In addition, the measurement times for the generation of a well re solved Raman image often are prohibitively long.

By exploiting nonlinear optical effects, novel microscopy tech niques have been developed over the last ten years to rapidly im age living cells in a chemically specific label free way. As a matter of fact, most non linear optical processes do not rely on the pres ence of a chromophore since a nonlinear signal is generated, with varying efficiencies of the process, in any polarizable medium, i.e. in any sample. This said, it is important to note that different sym metry restrictions and resonance conditions apply to different non linear effects. For example, second harmonic generation (SHG), that is the generation of light at exactly half the wavelength of the exciting light, has been used extensively for imaging collagen fibers $[5,6]$ since the formation of SHG signal reflects the local sym metry and order of the sample. Specifically, SHG is forbidden in iso tropic media, which causes the SHG signal to be suppressed in most of the liquid cell interior due to the random orientation of the molecules. Structures like collagen fibers, however, are or dered, allowing the coherent addition of the SHG from the individ ual non inversion symmetric molecules. Another non linear process used for label free optical microscopy is third harmonic generation (THG), producing light with exactly a third of the wave length of the exciting light. THG is created also in inversion sym metric structures, but due to the significantly different refractive index at the THG wavelength, the coherent superposition oscillates in space and is suppressed away from interfaces or structures hav ing sizes comparable to the excitation wavelength $[7,8]$. Since lip ids have a much higher nonlinear susceptibility $[9,10]$ for THG than water, this microscopy modality is useful for imaging lipid rich structures $[11,12]$. On the other hand, SHG and THG do not provide vibrational contrast, since the involved light frequencies are well above the vibrational frequency range. This situation changes when laser fields containing frequency differences in the vibra tional frequency range are used for excitation. By tuning the fre quency difference in the exciting light field to match the frequency of a molecular vibrational resonance, this resonance is driven coherently, such that the resulting Raman scattering of each molecule is mutually coherent. This leads to coherent Raman scat tering (CRS), in which the Raman scattering fields of all molecules constructively interfere, which results in a quadratic scaling of the scattered intensity with the number of molecules, as opposed to 
the linear scaling in spontaneous Raman scattering. High concen trations of molecules with similar vibrational resonances, as can be found in lipids, and especially in lipid droplets, therefore give a strong CRS signal. The most common implementation of CRS is to use two excitation frequencies, and to detect the frequency up shifted Raman scattering of the higher excitation frequency. called coherent anti Stokes Raman scattering (CARS). CARS micros copy has first been described in the early 80's [13], however for practical reasons it has found widespread application only in the last decade [14]. More recently also stimulated Raman scattering (SRS), which detects the CRS components spectrally overlapping with the excitation fields, has been implemented [15 17]. Similar to THG, CRS is a four wave mixing (FWM) process in which three exciting waves result in one signal wave. Differently from THG, however, CRS enables spectroscopic investigations, i.e. one can tune the frequency difference of the exciting lasers, which allows the identification of different chemical species according to their vibrational spectra. The advantages of CRS microscopy have soon become apparent such that over the last decade many different applications in biology have been published and dedicated micro scopes are now commercially available.

Most CRS applications so far have been aimed at label free imaging of lipids in a variety of samples from artificial model sys tems, to living cells and tissues to whole organisms. On the one hand this prevalence is due to the important important role of lip ids in biology. On the other hand, lipids exhibit favorable signal strengths in CRS experiments. CRS microscopy can therefore help to answer many open questions on lipid related processes inside cells and tissues, which due to the limitations and artifacts associ ated with fluorescence lipid staining have been left open. The abil ity of CRS microscopy to image lipids label free and with high spatial and temporal resolution in living cells offers a unique tool for the study of lipid biology. In this article, we will review the experimental implementations of CRS microscopy reported to date and their applications to image lipids in various systems from arti ficial membranes to living cells, tissues and model organisms. The paper is organized as follows. Section 2 covers the theoretical back ground of vibrational micro spectroscopy from linear to nonlinear effects, necessary for the understanding of CRS microscopy. Sec tion 3 discusses and compares different experimental implementa tions of CRS in terms of sensitivity limits and excitation and detection methods. An overview of the applications of CRS micros copy ranging from membrane model systems to live model organ isms is given in Section 4.

\section{Principles of vibrational micro-spectroscopy}

\subsection{Linear micro spectroscopy}

Let us recall some basic principles of vibrational spectroscopy, starting with the case of linear optical phenomena, namely infrared absorption and Raman scattering.

IR absorption originates from the motion of partially charged atoms in molecules. If the corresponding electric dipole moment changes during the vibration, then the transition is IR active. Homonuclear diatomic molecules, such as $\mathrm{N}_{2}$, have no partial charges and their vibrations are consequently not infrared active. Examples of simple IR active molecules are shown in Fig. 1. Carbon dioxide is a linear molecule with inversion symmetry and partial charges are indicated. The symmetric stretch does not result in a change in electric dipole moment and hence is not IR active, while the other two modes, the asymmetric stretch and the bend, are IR active. For water, a non linear molecule with reflection symmetry, all modes are IR active. In larger molecules, typically all modes are to some extend IR active due to missing symmetry.

In general, an $\mathrm{N}$ atomic non linear molecule has $3 \mathrm{~N} \quad 6$ vibrational normal modes. Therefore, in contrast to electronic
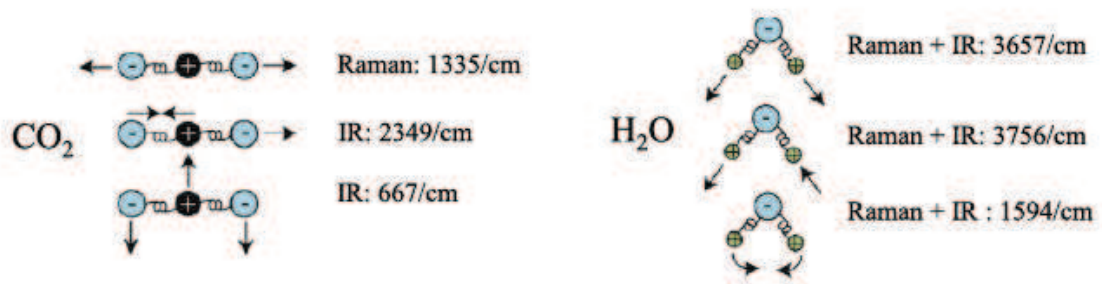

Fig. 1. Sketch of vibrational modes of $\mathrm{CO}_{2}$ (left) and $\mathrm{H}_{2} \mathrm{O}$ (right). The vibrational frequencies are given, together with their relevance in infrared absorbtion (IR) or Raman scattering. Partial charges of the atoms are indicated.

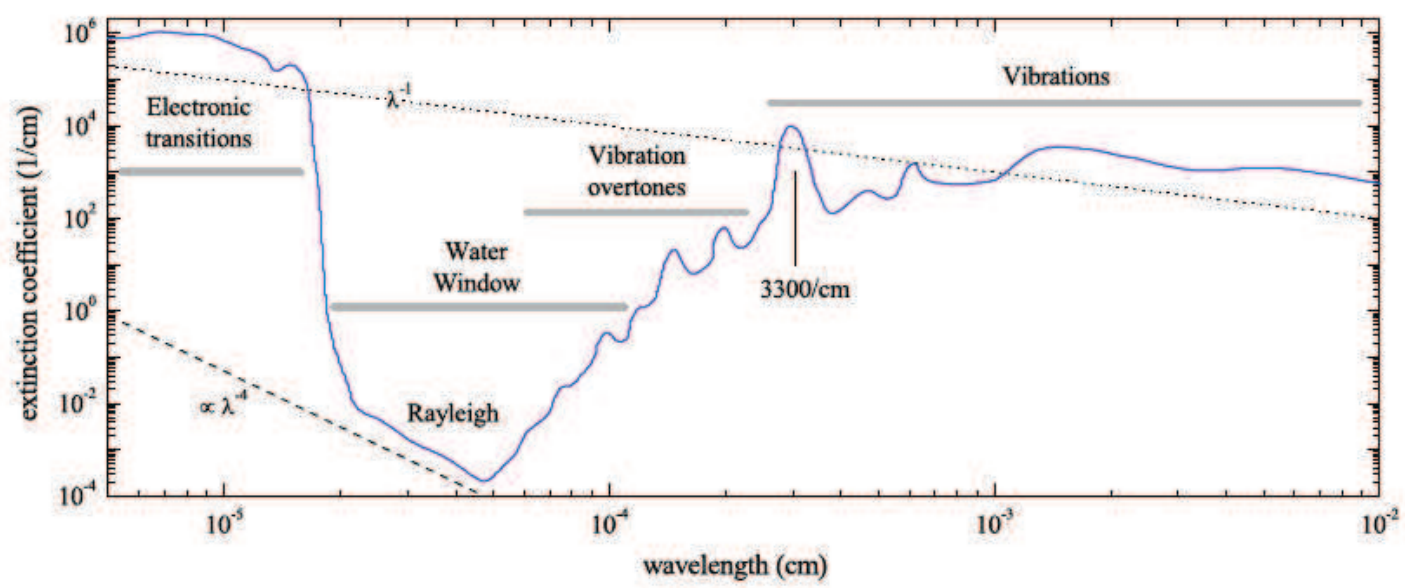

Fig. 2. Extinction coefficient of liquid water. The mechanisms relevant for the different wavelength ranges are labeled. The dotted line indicates an extinction coefficient equal to the inverse wavelength $\lambda^{1}$, and the dashed line indicates the scaling $\lambda^{4}$ of the extinction by Rayleigh scattering. 
absorption spectra, vibrational spectra show a large number of dif ferent bands. An example of an IR transmission spectrum is given in Fig. 3 for cholesterol $\left(\mathrm{C}_{27} \mathrm{H}_{46} \mathrm{O}\right)$, the principal sterol synthesized in cells and a building block of cell membranes. In organic mole cules, vibrations in two main spectral ranges can be distinguished. The high frequency range $27003600 \mathrm{~cm}^{1}$ (frequencies are given as wavenumbers, which are the inverse of the corresponding light wavelength in vacuum) is related to hydrogen stretching modes, with $\mathrm{C} \mathrm{H}$ bonds being at lower frequencies $\left(\leqslant 3100 \mathrm{~cm}^{1}\right)$ than $0 \mathrm{H}$ bonds. Lower frequencies $\left(<1800 \mathrm{~cm}{ }^{1}\right)$ relate to stretching modes of heavier atoms such as C, N, O but also deformation modes involving hydrogen. Strongly delocalised modes (e.g. breathing modes in the sugar ring of nucleic acids) give rise to vibrational resonances $<1000 \mathrm{~cm}^{1}$. The lower frequency range is commonly known as the fingerprint region, since it exhibits a characteristic band pattern which can be used to identify chemical compounds.

Although IR absorption is a powerful spectroscopic technique for chemical characterization, its application to cell microscopy has two main drawbacks. The first one is the spatial resolution which scales with the light wavelength $\lambda$. A resolution of in the or der of 10 micrometers over the typical vibrational spectral range $\left(1000 \mathrm{~cm}{ }^{1}\right.$ vibrational frequency equates to $\left.\lambda \quad 10 \mu \mathrm{m}\right)$ is not sui ted for subcellular imaging. The second drawback is the limited penetration depth by the absorption process itself in particular when taking into account that water, a dominant component of most living materials, has a strong IR absorption for $\lambda>3 \mu \mathrm{m}$ cor responding to an extinction length $<10 \mu \mathrm{m}$ (see Fig. 2).

Differently from the process of light absorption, in which light is converted into an internal excitation, light can also be scattered into a different optical mode. Vibrational Raman scattering may be regarded as an inelastic collision of light with a vibrating mole cule, where following the collision the light has a different fre quency. The energy difference between the incident and scattered photon equates the vibrational energy gained or lost by the molecule. Scattered light with higher (lower) frequency than the incident one is called anti Stokes (Stokes) component.

Compared to IR absorption, Raman scattering uses visible light. Hence, in Raman microscopy the spatial resolution is greatly im proved and equals that of confocal fluorescence microscopy. Selec tion rules for Raman scattering are different from those of IR absorption (see Fig. 1) since Raman scattering does not rely on the electric dipole moment from charge displacements. Instead, Raman activity of a transition requires a change in the molecular polarizability during the vibration (for a detailed account of Raman scattering theory and selection rules see Ref. [18]). Consequently the vibrational spectra of organic molecules detected by IR or Ra man techniques show the same resonances but with different weights. An example of a Raman spectrum of cholesterol is shown in Fig. 3. In comparison to the IR spectrum, the same resonances are visible, but with different relative strength.

A limitation of Raman spectroscopy is that tiny amounts of fluo rescent material can create a significant background, as the cross section for absorption is many orders of magnitude larger than for Raman scattering. For example, glass often used for mounting sam ples in optical microscopy is a disordered material with a tail of electronic states which creates a spectrally broad luminescence background. Fused silica (quartz) has a higher band gap and thus a lower density of localized states at a given wavelength, reducing this background. Using a pure crystalline material such as $\mathrm{CaF}_{2}$ avoids this tail of electronic states and results in a low lumines cence background. Typical values of Raman scattering cross sec tions are $10^{29} \mathrm{~cm}^{2}$ per vibrational mode, which is equivalent to a disc of $0.03 \mathrm{fm}$ diameter, about 50 times smaller than the nucleus of carbon. For comparison, absorption cross sections for electronic transitions are typically $10^{15} \mathrm{~cm}^{2}, 14$ orders of magnitude larger and equivalent to $0.3 \mathrm{~nm}$ diameter, about the diameter of the car bon atom. Due to the small cross section, Raman microscopy with a laser power of $10 \mathrm{~mW}$ at $532 \mathrm{~nm}$, limited by photodamage, re quires integration times of at least $0.1 \mathrm{~s}$ per point, and accordingly minutes to hours for images of $10 \times 10$ to $100 \times 100$ pixels, which is generally incompatible with live cell imaging.

\subsection{Nonlinear micro spectroscopy: coherent Raman scattering}

In linear Raman scattering, individual vibrational modes of mol ecules within the excitation volume are scattering the incident light incoherently with each other. Since thermally excited vibra tions have random phases, also the spontaneous Raman emission has a random phase. The scattered fields are thus interfering ran domly such that the time averaged intensity of the scattering scales linearly with the number of modes $N_{m}$ in the focal volume. If the scattering was coherent, the fields would constructively interfere and the scattered field would scale linearly with $N_{\mathrm{m}}$, with the intensity scaling as $N_{\mathrm{m}}^{2}$, a factor of $N_{\mathrm{m}}$ enhancement compared to the incoherent case. The typical focal volumes in high resolution optical microscopy are of the order of a femtoliter $\left(1 \mu \mathrm{m}^{3}\right)$, and a typical molecule volume is $1 \mathrm{~nm}^{3}$. Thus up to about $10^{9}$ molecules can fit into the focal volume, providing an intensity enhancement of up to $10^{9}$. Even without reaching such densely packed molecular arrangement, an enhancement in the $10^{6}$ range would allow for an increase in the imaging speed enabling video rate imaging with pixel dwell times of $100 \mathrm{~ns}$.

Coherent Raman scattering microscopy exploits such a coherent enhancement effect by driving the oscillation of modes using the

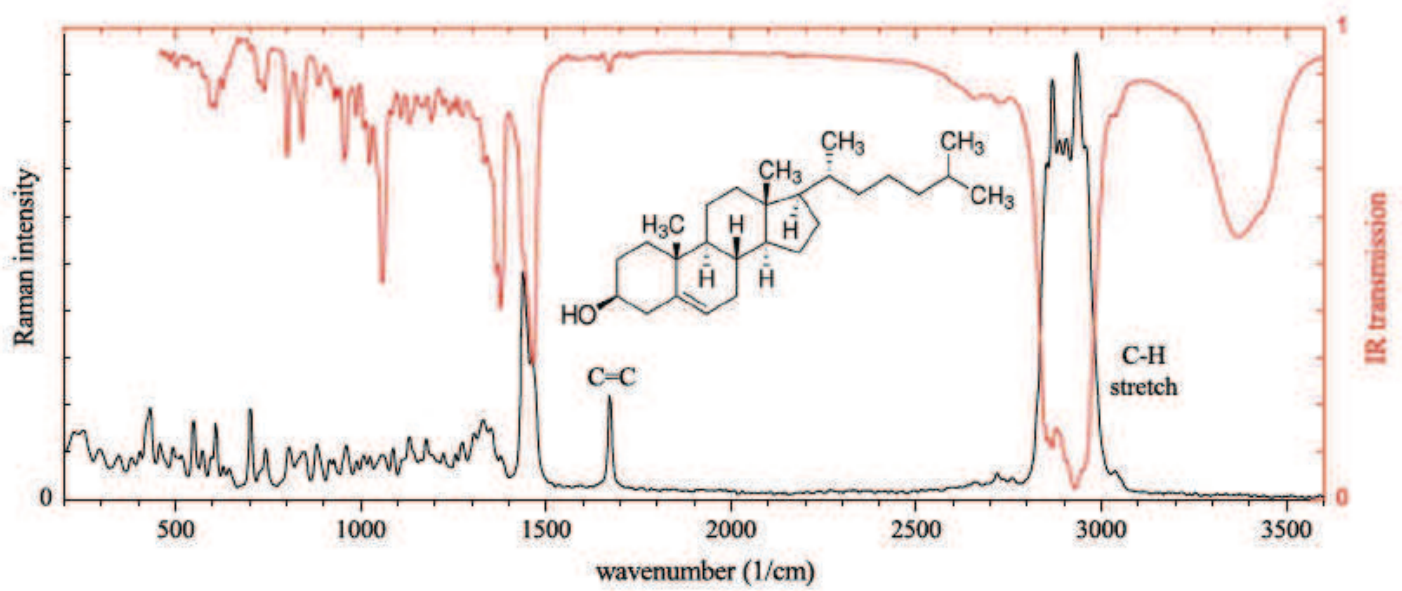

Fig. 3. Vibrational spectra of cholesterol having a chemical structure as shown. Raman scattering intensity (black) and infrared transmission (red) are shown [19]. 
interference between two light sources (called pump and Stokes). Light wavelengths are typically chosen in the so called biological window ( $\lambda \quad 6001300 \mathrm{~nm}$ ) i.e. the spectral range of minimum absorption from water (extinction length is larger than $1 \mathrm{~cm}$ ) and away from electronic absorption of most organic molecules. The frequency difference between pump and Stokes light fields (and hence the low frequency beating term of their interference) is then adjusted to be resonant to the molecular vibration of interest.

Without discussing the details of a mathematical description (which can be found in Ref. [20]) the coherent Raman process is de scribed by light induced transitions between molecular energy levels, as shown in Fig. 4a. A semiclassical picture is used in which light is a classical field and molecular vibrations are quantized lev els. Pump and Stokes fields ( $\mathcal{E}_{\mathrm{p}}$ and $\mathcal{E}_{\mathrm{S}}$ respectively) create, via a vir tual intermediate state, a coherence between the first two vibrational levels $\Psi_{0,1}$ of the electronic ground state $S_{0}$. For a given driving frequency, vibrational resonances at significantly higher frequency are providing a constant real background in the coherent Raman polarization. Furthermore, other third order nonlinearities (so called electronic contributions) are contributing to the CRS, as summarized in Fig. 4b. They have only virtual intermediate states and contribute to a non resonant real susceptibility $\chi_{e}$, which is also known as Kerr effect. If the upper intermediate state of this process is in resonance to e.g. an excited electronic state, two photon absorption occurs, which increases the photo damage significantly. The total CRS polarization can thus be written as a sum over all the vibrational resonant parts and an electronic con tribution which we will assume to be non resonant in the follow ing. This defines a susceptibility in frequency domain as

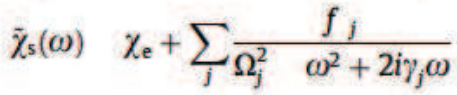

The sum runs over all vibrational modes numbered by $j$ and we have introduced the Raman oscillator strength $f_{j}$, the resonance fre quency $\Omega_{j}$ and the linewidth $\gamma_{j}$ of the $j$ th vibrational mode. In time domain, the susceptibility $\chi_{s}$ relates to the induced dipole moment $p$ of a molecule via

$p(t) \quad E(t) \int_{\infty}^{t} \chi_{s}\left(t \quad t^{\prime}\right) E^{2}\left(t^{\prime}\right) d t^{\prime}$

where $E$ is the real electric field amplitude. Substituting $\mathcal{E}_{\mathrm{p}}$ and $\mathcal{E}_{\mathrm{S}}$. oscillating at optical frequencies $\omega_{\mathrm{p}}$ and $\omega_{\mathrm{s}}$ respectively, into the field $E$ in Eq. (2) produces the different frequency components of CRS (see Table 1). In practical applications, $\mathcal{E}_{\mathrm{p}}$ and $\mathcal{E}_{\mathrm{S}}$ are trains of pulses to provide sizeable peak fields, needed to generate nonlinear effects, whilst maintaining moderate average powers to limit sam ple heating. In this case $\omega_{\mathrm{p}}$ and $\omega_{\mathrm{S}}$ indicated in Table 1 are their
Table 1

Components of the coherent Raman polarization induced by the pump and Stokes field $\mathcal{E}_{\mathrm{p}}, \mathcal{E}_{\mathrm{s}}$ with frequencies $\omega_{\mathrm{p}}$ and $\omega_{5}$.

\begin{tabular}{lll}
\hline Frequency & Acronym & Amplitude \\
\hline $2 \omega_{\mathrm{p}}-\omega_{\mathrm{S}}$ & CARS & $\mathcal{E}_{\mathrm{p}} \mathcal{E}_{\mathrm{p}} \mathcal{E}_{\mathrm{s}}$ \\
$\omega_{\mathrm{p}}$ & SRL & $\mathcal{E}_{\mathrm{p}} \mathcal{E}_{\mathrm{s}} \mathcal{E}_{\mathrm{s}}$ \\
$\omega_{\mathrm{S}}$ & SRG & $\mathcal{E}_{\mathrm{p}} \mathcal{E}_{\mathrm{p}}^{*} \mathcal{E}_{\mathrm{s}}$ \\
$2 \omega_{\mathrm{s}}-\omega_{\mathrm{p}}$ & CSRS & $\mathcal{E}_{\mathrm{p}} \mathcal{E}_{\mathrm{s}} \mathcal{E}_{\mathrm{s}}$ \\
\hline
\end{tabular}

center optical frequency. Note that due to the resonant nature of the vibrational parts in $\chi_{s}(\omega)$, information about individual modes is localized in frequency domain, while the non resonant electronic background is localized in time domain. Given that for an excitation consisting of pump and Stokes there are four CRS fields of similar amplitude, one can ask the question which one to detect, which we will discuss in the following section.

\section{Coherent Raman scattering: sensitivity limits, excitation and detection methods}

\subsection{CARS and CSRS}

CARS and Coherent Stokes Raman Scattering (CSRS, see Table 1) are free from excitation background since they can be spectrally well separated from the frequency of the exciting pump and Stokes pulses. They are typically 10 orders of magnitude weaker in inten sity than the excitation pulses, yielding for $10 \mathrm{~mW}$ average power in the excitation a CARS power of $1 \mathrm{pW}$, corresponding to $10^{6} \quad 10^{7}$ photons per second. These can be detected with sensitive detectors such as photomultipliers (PMTs) or avalanche photodiodes (APDs). If the CARS signal is spectrally resolved, also CCD Cameras can be used, which are however much slower to read out, typically milli seconds compared to nanoseconds for APDs and PMTs. Of the two signals, CARS is advantageous due to its shorter wavelength, at which these detectors typically have a higher quantum efficiency. Furthermore, incoherent background due to fluorescence and $\mathrm{Ra}$ man scattering is mostly present at longer wavelengths, spectrally overlapping only with CSRS.

When detecting the scattered intensity proportional to the modulo square of the detected field, the non resonant response $\chi_{e}$ interferes with the resonant response, giving rise to a complex spectral shape (see Fig. 5). Different methods to mitigate this issue have been demonstrated in the literature. One approach is to mea sure the CARS spectrum over a large vibrational range and using appropriate algorithms such as the maximum entropy method $[21,22]$ or the time domain Kramers Kronig method $[23,24]$ to re trieve from $\left|\chi_{s}(\omega)\right|^{2}$ the complex quantity $\chi_{s}(\omega)$, containing the real intermediate state: resonance \& memory

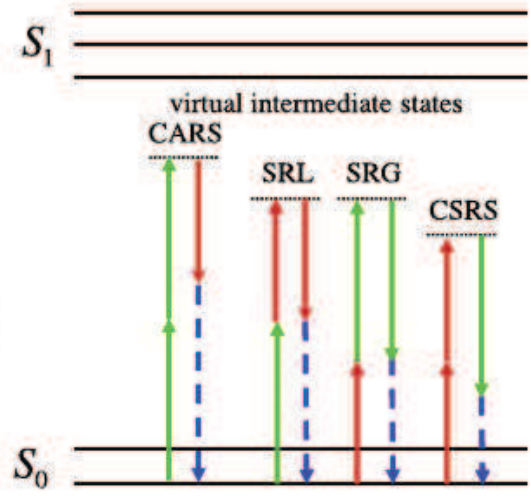

Fig. 4. Sketch of optical transitions in coherent Raman scattering. (a) Vibrationally resonant scattering, (b) Non-resonant scattering. $S_{0}$ : electronic singlet ground state, $S_{1}$ : first electronic singlet excited state of the molecule. 

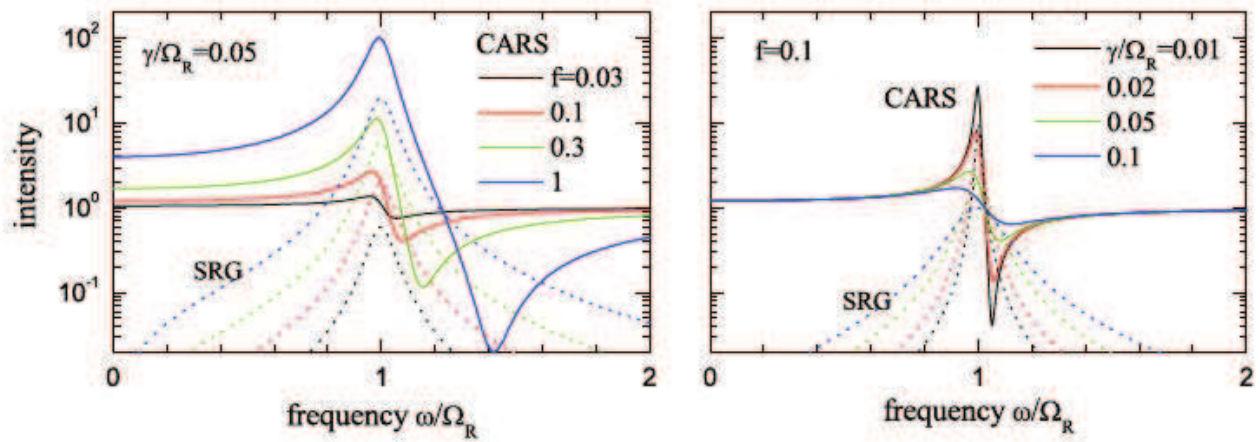

Fig. 5. Intensity of CRS signals as function of the detuning of $\omega \quad \omega_{p}-\omega_{s}$ for different Raman oscillator strengths $f$ and linewidths $\gamma$ as labelled. The CARS intensity $\left|\chi_{s} / \chi_{e}\right|^{2}$ and the SRG intensity $2 \Im\left(\chi_{\mathrm{s}} / \chi_{\mathrm{e}}\right)$ are scaled relative to the non-resonant background field. Note that typically the Stokes field $\mathcal{E}_{\mathrm{s}}$ is $10^{5}$ times larger than the non-resonant background, increasing the SRG intensity by the same factor.

resonances in the imaginary part. To measure the spectrum $\left|\chi_{s}(\omega)\right|^{2}$, one can either simultaneously excite a range of frequen cies using broad band pulses covering $2004000 \mathrm{~cm}^{1}$ vibra tional range (multiplex CARS), and use a spectrally resolved detection, or use a sequential approach tuning the center optical frequency of narrow band $\left(\sim 10 \mathrm{~cm}{ }^{1}\right)$ excitation by picosecond pulses (single frequency CARS). Note that typical vibrational reso nances of biomolecules in liquid have linewidths in the $10 \mathrm{~cm}{ }^{1}$ range, i.e. are matched to picosecond pulses. A limitation of the spectral retrieval approach is the acquisition speed, since a broad band spectral readout is typically in the millisecond time range. This is due to the limited light throughput of spectrometers and the finite acquisition speed of CCD cameras used in multiplex CARS, or the scanning time needed to sequentially cover large ranges in single frequency CARS. To increase the acquisition speed for real time imaging applications, one can resort to measuring only a small number of spectral points simultaneously. This has been done by a fast switch (in the $\mathrm{MHz}$ range) of the pump Stokes frequency difference hence exciting two (or more) vibrational fre quencies, and demodulating the CARS intensity detected by a fast and efficient single PMT. This method implemented in slightly dif ferent variants has been called in the literature frequency modu lated [25,26] (FM) CARS, Dual/Differential [27 29] CARS (D CARS, see Fig. 6), and Quadruple [30] CARS.

An alternative approach to reduce the influence of the non res onant response in the detected CARS intensity uses the vectorial polarization of light. The resonant CARS signal typically has a non isotropic Raman susceptibility characteristic for the specific resonance, such that by choosing specific excitation polarizations of pump and Stokes fields, the resonant and non resonant parts in CARS have different polarization directions [ 31 33]. Detecting the field polarized orthogonal to the non resonant signal elimi nates the latter. However, this technique suppresses also the reso nant intensity by typically an order of magnitude, and the depolarization depends on the molecular vibration, complicating the analysis of the signal. Furthermore, birefringence in the sample or in the optics can give artifacts by altering the polarization of the non resonant contribution.

Propagation effects of the CARS signal [32] can be used to sup press background from homogeneous media, similar to dark field contrast microscopy. CARS created by a bulk medium has a propa gation direction given by the wavevector conservation of the four wave mixing process, namely $\mathbf{k}_{\text {CARS }} \quad 2 \mathbf{k}_{\mathrm{p}} \quad \mathbf{k}_{\mathrm{S}}$ with $\mathbf{k}_{\mathrm{CARS}}, \mathbf{k}_{\mathrm{p}}$, and $\mathbf{k}_{\mathrm{S}}$ being the wavevectors of CARS, pump and Stokes fields respec tively. Due to the dispersion of the refractive index, for parallel beams there is a wave vector mismatch limiting the interaction length. Typically, for microscopy applications one focuses the pump and Stokes beams onto the sample using a high numerical aperture (NA) microscope objective in order to have a small focal volume and hence a good spatial resolution. In this case, the direc tions of $k_{\mathrm{p}}$ and $\mathrm{k}_{\mathrm{s}}$ are distributed over a range in the forward direc tion, such that the combination of wavevector directions and the limited interaction lengths given by the axial focus size allow to satisfy the wavevector conservation. The resulting directional range of $\mathbf{k}_{\text {CARS }}$ is centered in forward direction and distributed over a somewhat smaller solid angle [32] than $\mathbf{k}_{\mathrm{p}}$ and $\mathbf{k}_{\mathrm{s}}$. On the other hand, if the medium is spatially structured, $\chi_{\mathrm{s}}$ is distributed over a range of wavevectors which are convoluted with the excitation directions and extend the wavevector distribution of the signal. Detecting the CARS signal at wavevectors created outside the range for a bulk sample can thus be used to eliminate the contribution of the bulk like material. Therefore, if the sample investigated con sists of a small volume fraction of sub wavelength sized structures with strong resonant signal surrounded by a bulk like non reso nant medium, this method is beneficial. Most noted implementa tions are epi collection [32] and wide field CARS [34 36] which is a non scanning technique allowing for single shot image acqui sition. Furthermore, by modifying the angular phase distribution of pump and Stokes, the bulk CARS signal can be suppressed in for ward direction [37]. In principle the most straightforward method to distinguish the resonant contribution in $\chi_{s}$ is to actually measure the CARS signal in amplitude and phase, which however requires an interferometric detection which adds to the complex ity. This approach was shown in a modulated heterodyne detection scheme $[38,39]$, allowing for a fast single channel detec tion, or in a multiplex CARS scheme by spectral interferometry $[40,41]$.

\subsection{SRL and SRG}

Stimulated Raman loss (SRL) and stimulated Raman gain (SRG) processes, generally called stimulated Raman scattering (SRS), give rise to signals spectrally superimposed to the excitation fields (see Table 1), which have typically $10^{4} \quad 10^{6}$ times higher amplitude $[16,17]$. The excitation fields have a fixed phase relation to the sig nals, and thus represent phase stable homodyne fields for the SRG and SRL signals. The imaginary part of $\chi_{s}\left(\mathfrak{I}\left(\gamma_{s}\right)\right)$ creates an in phase signal and the intensity change of the transmitted excitation field is therefore proportional to $\mathfrak{J}\left(\chi_{s}\right)$ (see Fig. 5), which is directly the resonant part (the non resonant term $\chi_{\mathrm{e}}$ has no imaginary part) and proportional to the spontaneous Raman intensity spectrum (in case of exponential dephasing). The combined effect of SRL and SRG transfers photons from the pump to the Stokes beam under the creation of a vibrational excitation receiving the photon energy difference $h\left(\omega_{\mathrm{p}} \omega_{\mathrm{s}}\right)$, hence it requires a vibrational resonance. Moreover, incoherent backgrounds in the detection of these signals are negligible due to large homodyne gain. However, the small change in intensity of the transmitted excitation by the SRL/SRG 
(a)

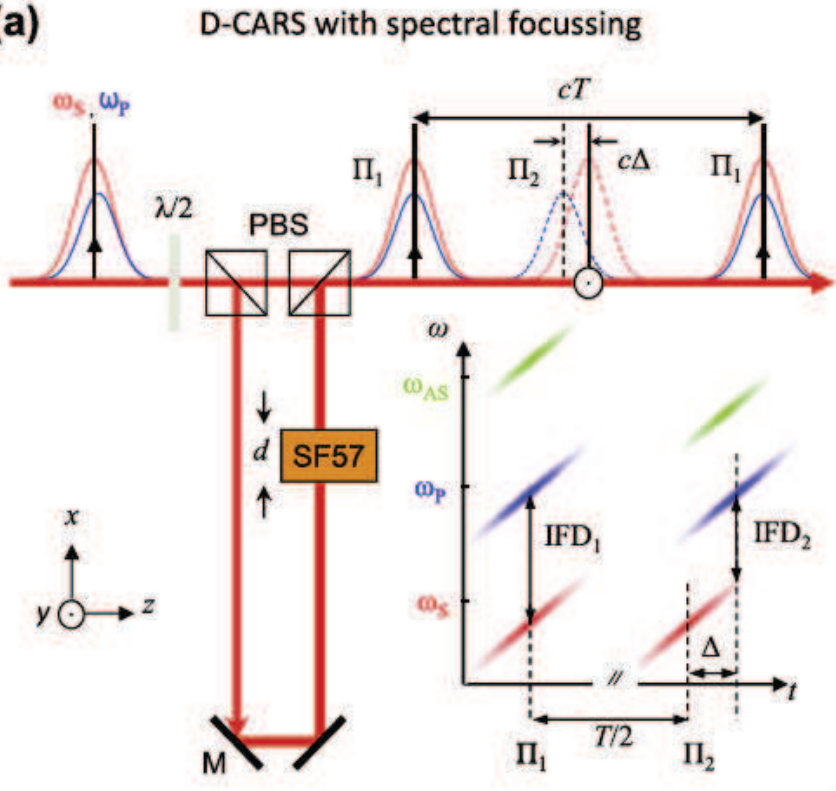

(b)
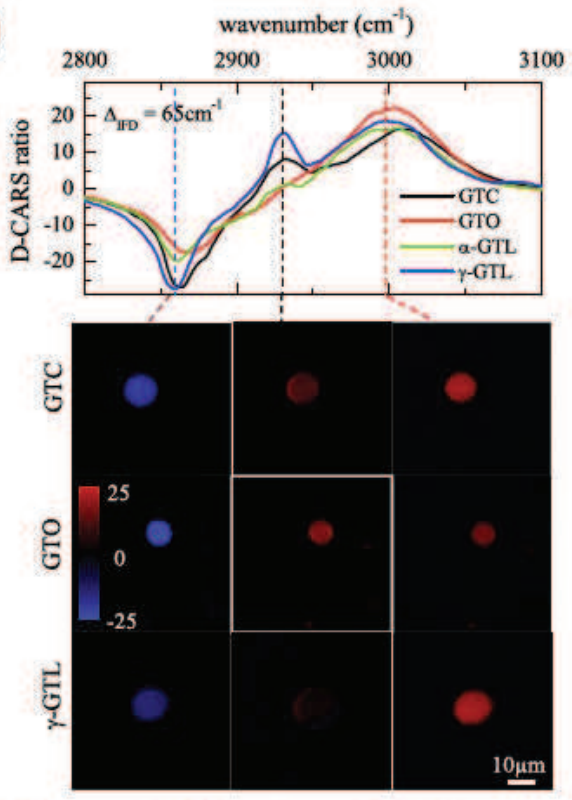

(a) $2850 \mathrm{~cm}^{-4} \quad$ a $2900 \mathrm{~cm}^{-1} 29 \mathrm{~cm}^{-1}$
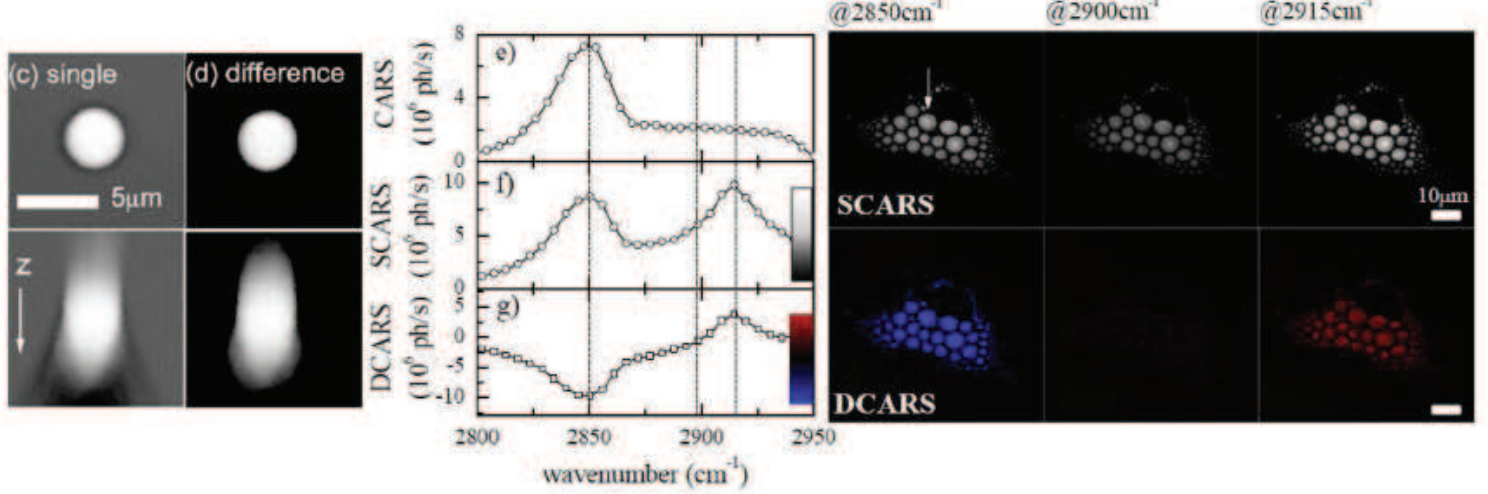

Fig. 6. (a) Sketch of differential/dual CARS (D-CARS) with spectral focussing. A pump-Stokes pulse pair $\Pi_{1}$ is split into two orthogonally polarized pairs using a half-wave plate $(\lambda / 2)$ and a polarizing beam-splitter (PBS). The second pair $\Pi_{2}$ travels through a thin SF57 glass block which introduces a delay time between Stokes and pump pulses due to their different group velocity and in turn results in the excitation of a vibrational resonance at a different frequency (via spectral focussing) compared to $\Pi_{1}$ (adapted from Ref. [27]). (b) Example of D-CARS spectra and images showing the ability to distinguish lipid droplets made from different lipid types (GTC: glyceryl tricaprylate, GTO: glyceryl trioleate, GTL: glyceryl trilinolenate, Adapted from Ref. [29]. (c, d) In plane $(x, y)$ and in depth $(x, z)$ images of a polystyrene bead in agarose gel (on a logarithmic gray scale over 2 orders of magnitudes) using single-frequency CARS at the resonance frequency of the $\mathrm{CH}$ aromatic band at $3050 \mathrm{~cm}{ }^{1}$ and D-CARS at the difference between the in-resonance and out-of-resonance ( $3110 \mathrm{~cm}$ ') frequencies, showing the ability of D-CARS to suppress the non-resonant background (adapted from Ref. [27]). (e-g) CARS, Sum-CARS (SCARS) and D-CARS spectra and corresponding images in stem-cell derived human adipocytes (adapted from Ref. [42])).

process, typically in the order of $10^{5}$, is superimposed to classical laser noise of the excitation and modulation by the spatially vary ing sample transmission during scanning. To allow discrimination of the signal in this condition, one can modulate the amplitude of the non detected excitation field which modulates the signal accordingly (see Table 1). Using an excitation modulation in the $110 \mathrm{MHz}$ range $[16,43,44]$ allows one to suppress classical laser noise and microphonics. The modulation of the sample transmis sion has a frequency cutoff given by the scan speed and the resolu tion. Allowing for several excitation modulation periods per pixel, and a pixel size half the spatial resolution for Nyquist sampling. this frequency cutoff is significantly below the excitation modula tion frequency and can thus be suppressed. However, in a modu lated technique the signal is created only during half of the total measurement time, reducing the efficiency. On the other hand, photodiodes with a quantum efficiency $(Q E)$ above $80 \%$ can be used for detection, roughly a factor of two larger than the QE of PMTs used for CARS.

Similar to FM CARS or D CARS, it is possible also in SRS to mea sure a few vibrational frequencies simultaneously by modulating and detecting at different modulation frequencies [45]. The Stokes pulse can also be spectrally shaped to measure the projection of the SRL response onto non trivial vibration frequency distributions [46]. The implementation of multiplex SRS using broadband laser sources and spectrally resolved detection is possible in principle, but is presently limited [47] by the multiplex detectors available, due to the high power and high frequency modulation to be de tected. Typical line scan CCD cameras which could be used for spectrally resolved detection have a pixel read rate of $100 \mathrm{MHz}$ and a full well capacity of $10^{5}$ electrons, and thus can detect about $2 \mu \mathrm{W}$, which is 34 orders of magnitude less than the excitation powers. Furthermore, spectra can be read with a rate of about $100 \mathrm{kHz}$, allowing for a modulation frequency 12 orders below the one used for fast scanning. These limits are due to the design of these cameras and can be overcome in the future within the same basic silicon technology by increasing full well capacity and allowing for on chip modulation. Multiplex SRS images are presently taken sequentially by tuning the frequency of the modu lated excitation beam with step times down to the millisecond range $[48,49]$, 
Another notable difference between SRS and CARS is that wave vector conservation is respected by definition in the process $\left(\begin{array}{llll}\mathbf{k}_{\mathrm{SRG}} & \mathbf{k}_{\mathrm{p}} & \mathbf{k}_{\mathrm{p}}+\mathbf{k}_{\mathrm{S}} & \mathbf{k}_{\mathrm{s}}\end{array}\right)$. Owing to the third order nonlinearity of the process the point spread function of the SRS is smaller than the one of the exciting beam such that the emitted signal has a wider directional range.

Without entering into detailed derivations (which can be found in Ref. [20]) it is important to point out that CARS and SRS have similar signal to noise ratio limits, basically because the related fields are of similar amplitude (see Table 1).

\subsection{Coherent control in CRS}

Coherent quantum control techniques such as Rabi oscillations or adiabatic passage have been proposed in the literature [50] to improve certain aspects of the CRS contrast. However, to avoid sample damage by avalanche breakdown or multiphoton ioniza tion [51], CRS is typically in the small perturbation regime, i.e. pump and Stokes pulses drive the vibrational coherence well be low the level of a full coherent population of the excited vibrational state (see Fig. 4a). Such coherent quantum control techniques are therefore not applicable for typical biological samples.

\subsection{Excitation schemes of CRS}

As already partly introduced in Section 3.1, various excitation schemes for CRS have been proposed in the literature, using broad band femtosecond pulses or narrow band picosecond pulses. Here we will discuss the advantages and limitations of the different excitation schemes in more detail.

\subsubsection{Single frequency excitation}

Single channel detection is easy to implement in commercially available microscopes and can be fast with pixel times down to the sub microsecond range, enabling video rate imaging [52,44]. Since the information about individual vibrations is localized in fre quency domain $\chi_{\mathrm{s}}$, it is advantageous to measure CRS at a specific drive frequency. The best compromise between signal strength and background suppression $[53,54,28]$ is achieved using a pulse dura tion in the excitation which matches the dephasing time of the vibration, typically in the picosecond time range. An excitation scheme with pump and Stokes pulses of picosecond duration was the first one used [14] and is still popular [55] due to its conceptual and practical simplicity. The related spectrogram is shown in Fig. 7 on the right. However, a practical issue with this approach is that for other multi photon contrasts, such as two photon fluorescence or second harmonic generation which are valuable to access in the same instrument, $100 \mathrm{fs}$ pulses are better suited since for a given average power their intensity typically scales inversely with the pulse length down to below $100 \mathrm{fs}$.
An alternative approach, named spectral focussing [56], uses femtosecond pump and Stokes pulses, linearly chirped with an equal slope. In this way the instantaneous frequency difference (IFD) between them (which determines the driving frequency via the interference of pump and Stokes in CRS) is constant, and the spectral bandwidth of the excitation is given by the inverse of the chirped pulse duration which can easily reach picosecond val ues (see also Fig. 6). The related spectrogram is shown in Fig. 7 in the middle. Furthermore, since the IFD can be tuned within the pulse spectral width by changing the delay time between pump and Stokes pulse, it allows spectroscopy by simply moving a delay line without the need for laser wavelength tuning. Using broad band Stokes pulses created by a fibre continuum [57] or a sub $10 \mathrm{fs}$ laser [58,42], a large vibrational frequency range of 1000 $4000 / \mathrm{cm}$ can be accessed. Moreover, simultaneous CARS/TPF and SHG excitation and detection is possible with the same laser source. Noticeably, in Ref. [42] the optimum chirp requirement for CARS together with the shortest (hence unchirped) pulse dura tion requirement for TPF/SHG were met simultaneously owing to the large spectral range of the 5 fs laser source available and the selection of fully separated portions of the laser spectrum to gen erate pump, Stokes and two photon excitation pulses.

\subsubsection{Single time excitation}

The counterpart of single frequency excitation is single time excitation, which is impulsively exciting the system, and probing $\chi_{s}(t)$ at $t \quad 0$. The related spectrogram is shown in Fig. 7. This is efficient in the simultaneous drive of many vibration, but ineffi cient in the readout. Specifically, the response is largely affected by the non resonant $\chi_{e}$, which has no information concerning the vibrational resonances and is instantaneous in time domain com pared to the resonant part which decays with the time scale of the vibrational dephasing time hence in the picosecond range. To gain more resonant information, two [59] or multiple [60] pulses of adjustable time delay $\tau$ have been used, where the CRS signal of the second pulse has a component $\chi_{s}(\tau)$, and the delay is scanned to measure $\chi_{s}$ which is then converted to $\chi_{s}$ by Fourier transformation. Generally this is a slow technique, not well suited for imaging. However, due to the possibility of reaching long delay times, the vibrational spectrum can be measured down to small frequencies and with a high spectral resolution.

\subsubsection{Multiplex excitation}

In multiplex excitation a spectrally broad pump pulse and a spectrally narrow probe pulse are used to excite a range of vibra tional resonance frequencies simultaneously and read them out with high spectral resolution. Historically the first implementation used a combination of a picosecond and a femtosecond Ti:Sapphire lasers [61] for pump and Stokes pulse, respectively, yielding a fre quency time spectrogram as shown in Fig. 8a. The Stokes pulse is
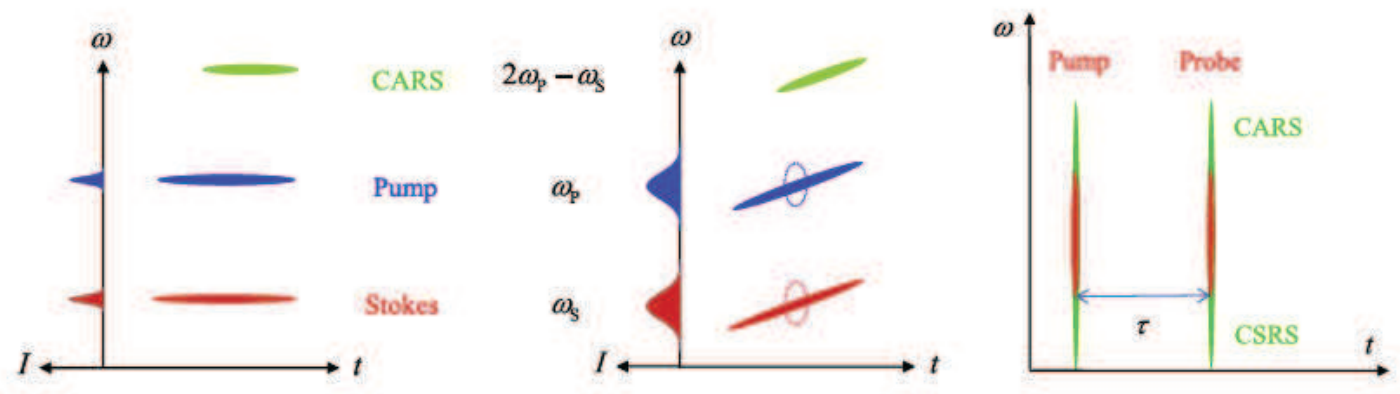

Fig. 7. Sketch of spectrograms in single frequency and single time CRS. The contributions of pump and Stokes excitation and CARS are labeled, and the excitation spectral intensity $I$ are shown. Left: chirp free excitation. Middle: Chirped excitation for spectral focussing. The dotted circles indicate the spectrograms for un-chirped pulses of the same spectrum. Right: Single time CRS. The pump and probe excitation separated in time by $\tau$, and the CARS and CSRS signal are indicated. 
(a) $\omega$

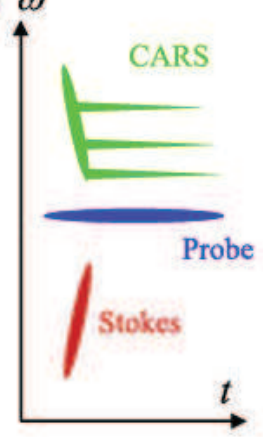

(b) $\omega$

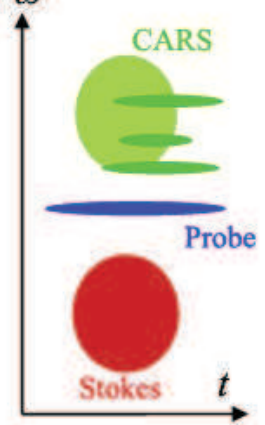

(c)

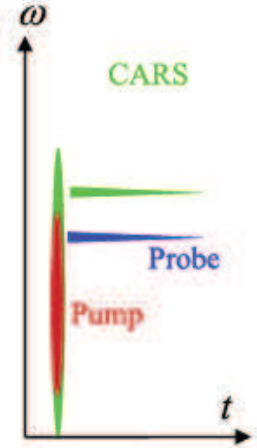

(d) $\omega$

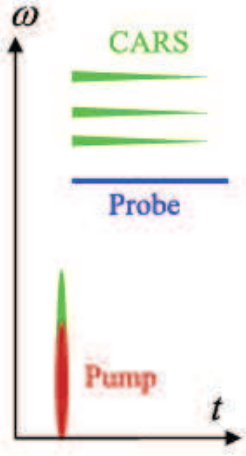

Fig. 8. Sketch of spectrograms in multiplex CRS. The contributions of pump and Stokes excitation and CARS are labeled. (a) coherent femtosecond Stokes and picosecond pump pulses (b) incoherent picosecond Stokes and picosecond pump pulses. (c) Coherent sub 10 fs pump pulse, picosecond probe pulse created by spectral filtering of pump. (d) 8 fs pump pulse at 1-1.5 $\mu \mathrm{m}$ wavelength, and frequency doubled picosecond probe pulse at $775 \mathrm{~nm}$ [66].

typically chirped by the dispersion of the microscope optics. Its interference with the pump pulse drives the vibration, which is subsequently probed by the pump pulse. This method has been widely used in the literature $[62,22]$, but a femtosecond picosec ond dual laser system is complex and costly. It can be simplified by using a single $\sim 100$ fs laser, which is spectrally filtered to provide the picosecond pulse while the remaining spectral compo nents are used for continuum generation in an optical fibre [63] to provide a broad band Stokes pulse. Alternatively, one can also use picosecond pulses from simpler laser systems such as Nd:YVO4, and continuum generation in a fibre for the Stokes pulse $[64,65]$. Noticeably, this continuum is incoherent since its generation in volves stimulated Raman gain and soliton instabilities, and the resulting spectrogram is shown in Fig. 8 b. The compromised time ordering leads to a somewhat weaker CRS, and the intensity fluctuations of the continuum are adding noise to the signal.

Another approach is to use a single femtosecond source of 5 20 fs duration, having sufficient bandwidth to excite the relevant vibrations, and spectrally shape the pulse to create a longer probe pulse on the short wavelength edge. The corresponding spectro gram is shown in Fig. 8c. The CRS is then detected by a multiplex spectral detector. The spectral shaping can be done by a liquid crystal based grating shaper demonstrated for CARS [67] and SRG/SRL [68], or with a notch filter demonstrated in CARS [69].

An alternative recent approach uses a sophisticated fibre laser system [66]. A 200 fs Erbium fibre oscillator at a wavelength of $1.5 \mu \mathrm{m}$ is split and amplified. One part is frequency doubled to pro duce picosecond probe pulses at $775 \mathrm{~nm}$, the other part is spec trally broadened in a fibre and compressed to deliver $8 \mathrm{fs}$ pulses at $10001400 \mathrm{~nm}$, providing the pump pulses. The related spectro gram is shown in Fig. 8 d. Due to the rectangular shape of the probe pulse, its rising edge can by placed a delay time $\tau$ after the pump, suppressing the instantaneous response $\chi_{e}$ by measuring $\chi_{s}(t)$ for $t>\tau$. The rather long wavelength of the pump pulse is also advan tageous due to the small glass dispersion in this wavelength range, reducing the complexity of chirp compensation of the pulse in the microscope focus, and the reduced photo damage being below the threshold for three photon absorption in typical biological mate rial such as proteins, lipids and sugars.

\section{Applications to lipid systems}

Coherent Raman micro spectroscopy has already found a vari ety of applications ranging from material science to in vivo investi gations of live mice. The CRS signal from lipids is particularly strong due to the large number of identical bonds in the focal volume providing a large coherent enhancement as discussed in Section 2.2. Investigations of lipid rich materials have conse quently been an early focus of CRS microscopy applications.

\subsection{Membrane model systems}

Soon after the development of the modern CARS microscope [14], experiments on membrane model systems have been published. These systems, which include multilamellar vesicles, small, large and giant unilamellar vesicles (MLV, SLV, LUV, and GUV, respec tively) and deposited bilayers are attractive samples for CARS microscopy, since their chemical composition is well defined. This allows subtle changes in the spectra, which can occur for example by temperature induced phase transitions, to be monitored directly. While a huge number of fluorescence microscopy based work has been published on these systems, label free CARS microscopy is attractive here, since it precludes the occurrence of labeling artifacts.

The first CARS microscopy work on multilamellar vesicles intro duced multiplex CARS microscopy as a new variant of this approach [70]. As explained in 3.4.3, multiplex CARS microscopy offers the possibility to record a CARS spectrum over a certain vibrational fre quency range at every image pixel. In this respect, the approach is similar to spontaneous Raman micro spectroscopy, where also a whole vibrational spectrum is recorded at every sample position. However, multiplex CARS spectra and Raman spectra cannot be compared directly since CARS spectra are affected by the non reso nant background (see Section 2.2). In Ref. [70] the authors show that monitoring skeletal C Cstretch vibrations of the lipid acyl chains at approximately $1100 \mathrm{~cm}^{1}$ can be used to differentiate between re gions onvesicles, or whole vesicles, in gel phase or liquid crystalline phase. Specifically they show that it is possible to distinguish DSPC and DOPC vesicles which have high and low phase transition tem peratures, respectively. The multilamellar vesicles investigated in Ref. [70] were approximately $2 \mu \mathrm{m}$ in diameter, resulting in an exci tation volume which is completely filled with lipids. The situation changes drastically, when unilamellar vesicles are investigated. For these, the number of molecules in the focus is three to four orders of magnitude smaller. Given that the CARS intensity depends qua dratically on the number of scattering molecules, this corresponds to a CARS signal decrease of six to eight orders of magnitude. Detec tion of a single lipid bilayer therefore pushes CARS microscopy to its sensitivity limits. The first successful detection of lipid bilayers by CARS microscopy was reported by Potma and coworkers on DOPC DOPG GUVs and on erythrocyte ghosts (Fig. 9) [71]. In these experi ments, the authors used single frequency CARS measurements both in a transmission type geometry and a backscattering (epi) detection configuration. They show that in the epi detection the signals can be amplified by non resonant signal from the glass water interface, which facilitates the detection, but also leads to interference effects in the images. In imaging lipid bilayers with CARS microscopy, polar ization effects occur due to the orientational order of the lipid chains, resulting in a higher CARS intensity [71] for a linear excitation polar ization in the bilayer plane, i.e. orthogonal to the lipid chains. 


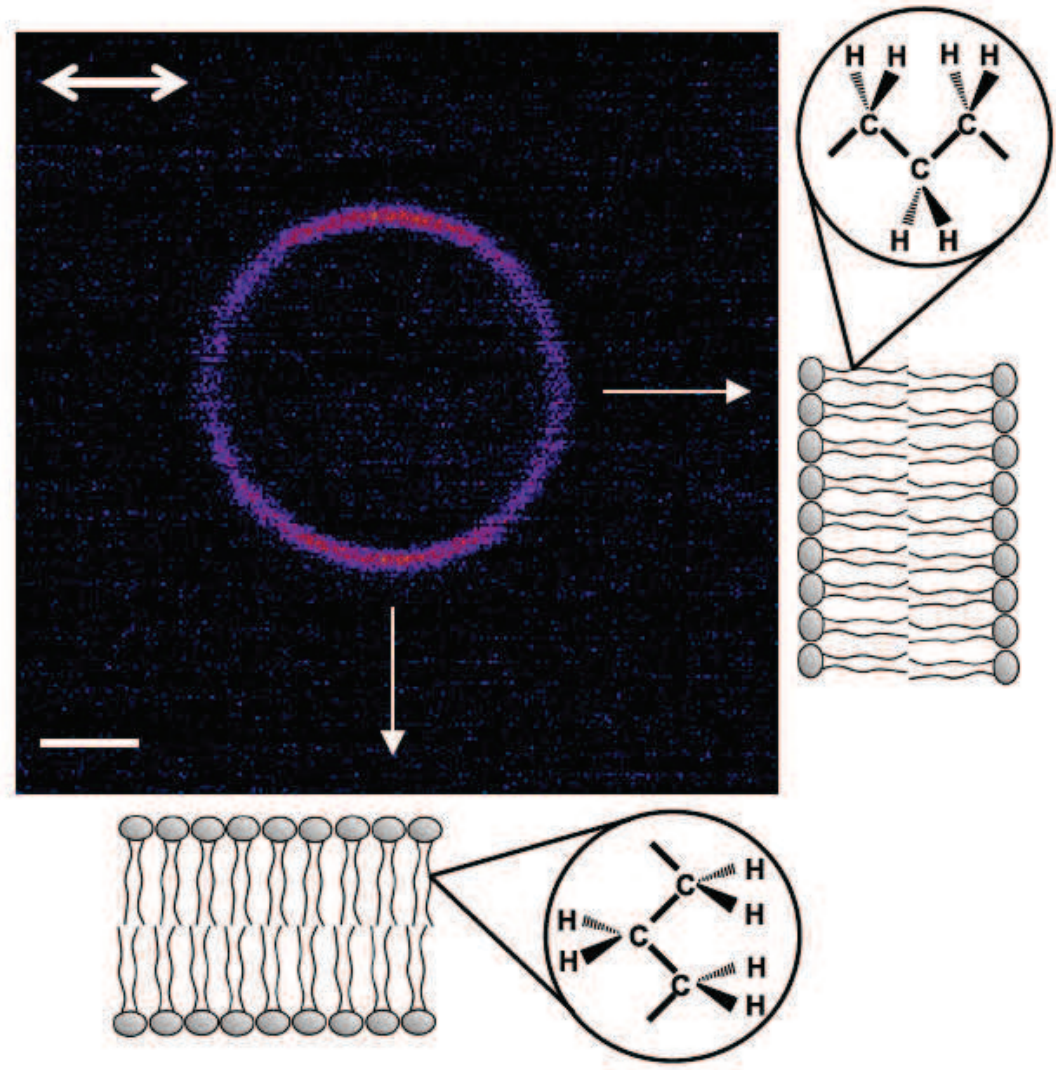

Fig. 9. Forward-CARS image of an erythrocyte ghost. The image was taken in the equatorial xy plane of the vesicle at a Raman shift of $2845 \mathrm{~cm}{ }^{1}$. The arrows point toward the orientation of the bilayer, giving rise to a higher CARS intensity (top and bottom) when aver aged C-H bond orientation is parallel with the incident beams (double arrow), and a lower CARS intensity (left and right) when C-H bond orientation is perpendicular to the incident polarization. Scale bar is $2 \mu \mathrm{m}$. Image size is $256 \times 256$ pixels and pixel dwell time is $1 \mathrm{~ms}$. Adapted from [71].

Following these initial proof of concept investigations, CARS microscopy has been used to investigate structures and their dynamics in various membrane models. It has been shown earlier for spontaneous Raman microscopy, that chain order and packing as well as the presence of gauche configurations can be analyzed by rationing intensities for symmetric and antisymmetric $\mathrm{CH}_{2}$ stretch vibrations and of $\mathrm{CH}_{2}$ and $\mathrm{CH}_{3}$ vibrations [72,73]. Similar work was possible using CARS microscopy, with the advantage that meaningful spectra with sufficient signal to noise ratios could be obtained within $20 \mathrm{~ms}$ as compared to $120 \mathrm{~s}$ for the spontaneous Raman measurements [74]. In order to improve the CARS micros copy sensitivity, the authors again used a multiplexing scheme, with the advantage that the non resonant background from the environment is amplifying the resonant signal [62].

While CARS spectra can be acquired by either scanning the excitation frequency or by using the multiplex approach (see Sec tion 3.4), the interpretation of the spectra is non trivial. As dis cussed in Section 2.2 the CARS spectrum is composed of a resonant and a non resonant part. The CARS intensity spectrum therefore reflects the interference term between the resonant and non resonant parts. Depending on the relative strengths of both components with respect to each other, this can result in CARS lineshapes, which are different from those of spontaneous Raman spectra. Quantitative interpretation of CARS spectra re quires the knowledge of the non resonant signal alone and the application of appropriate algorithms in order to derive the reso nant spectrum (see Section 3). While this makes a spectral analysis cumbersome, the interference term can also serve as an inherent signal amplification. The usefulness of these approaches has been demonstrated in studies of the main lipid phase transition in DMPC LUVs and SUVs [22]. In this phase transition from the solid phase to the liquid crystalline phase, the rotational and translational mobil ity of the lipids changes significantly. In addition, an increase in trans gauche isomerizations is observed. The intensity rationing of different $\mathrm{CH}$ stretch vibrations (after retrieval of Raman like spectra from CARS intensity spectra) was used to quantify the changes and follow the phase transition as the temperature is changed [22].

Besides spectral information, insights into the sample structure can be gained by exploiting the polarization properties of the exci tation light and the sample (see also Section 3). Raman scattering for a given molecular vibration is described by a scattering tensor. This means that the observed Raman and CARS signals have a well defined polarization which depends on the excitation polarization, the tensor and the orientation of the molecules. Since symmetry considerations simplify the tensor, experiments in which the exci tation and the detection polarization are varied can be used to draw conclusions about the orientation of the chains. This ap proach allows the quantification of changes in the acyl chain ordering in MLVs composed of different phosphatidylcholines upon addition of small amounts of cholesterol. Significant in creases in ordering have been observed for amounts of approxi mately $10 \mathrm{~mol} \%$. [75].

CARS microscopy has also been used in a number of investiga tions on phase segregation and domain formation in mixed com pound systems. Most often, deuterated compounds are used in these experiments for the preparation of the vesicles and planar bilayers containing lipid mixtures. The advantage of this approach is that $\mathrm{CD}$ stretch vibrations have frequencies around $2100 \mathrm{~cm}$. hence are well distinguished from the $\mathrm{CH}$ stretch around $2900 \mathrm{~cm}^{1}$ and fall into a spectral range void of common reso nances, specific for rarely occurring groups such as $\mathrm{C} \mathrm{N}$ or $\mathrm{C} \equiv \mathrm{C}$. 

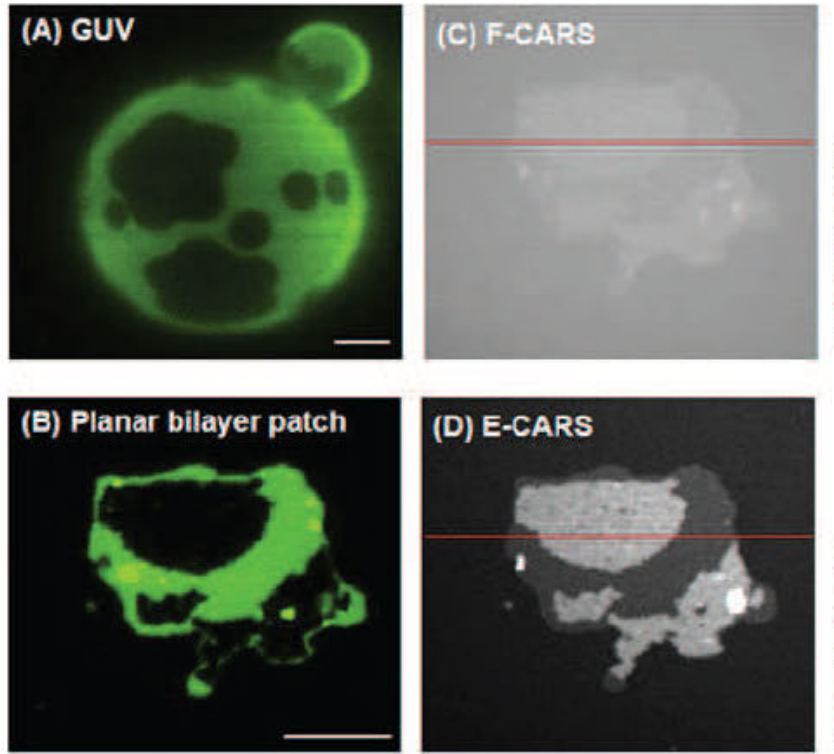
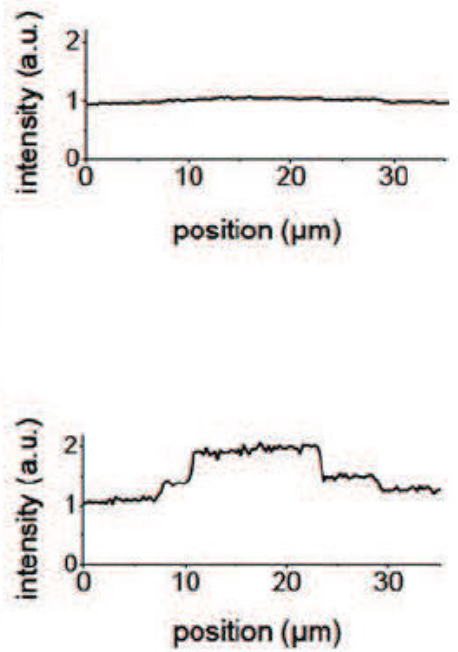

Fig. 10. (A) Fluorescence image of $1: 1$ DOPC/DPPC GUVs labeled with $0.4 \%$ BODIPY PC (B) Fluorescence image of a bilayer patch of a $1: 1$ mixture of DOPC and DPPC. (C) and (D) CARS images of the bilayer patch shown in (B) detected in forward-and in epi-direction, respectively. In this case, the contrast in the epi-image is better as in the forwardimage, since the bilayer is much thinner than the excitation wavelength. The intensity profiles along the red lines are shown below the images. The CARS images were averaged over 40 frames with an acquisition time of 0.44 s for each frame of $256 \times 256$ pixels. The pump and Stokes powers at the sample were 3.0 and 1.4 mW, respectively. Bar $10 \mu \mathrm{m}$. Adapted from [77].

It is therefore straightforward to isolate a specific lipid from a mix ture if that lipid is deuterated. CARS microscopy experiments of phase segregation were first done on mixed DOPC/[D70]DSPC GUVs in which only the [D70]DSPC phase was monitored using CARS microscopy [76]. For the visualization of the DOPC phase, $1 \%$ of fluorescently labeled DPPE was added and detected using simultaneous two photon. Combined two photon fluorescence and CARS experiments of this type are easy to implement, since they require only the addition of a second detection channel for a different spectral window. Monitoring of domain formation is also possible solely based on CARS microscopy by using deuterated and non deuterated lipids and measuring CARS at the CD and at the $\mathrm{CH}$ vibrations. The dependence of the CARS signal on the num ber of scattering molecules also enables the monitoring of different domains in lipid bilayers. This is possible if the domains differ in density of $\mathrm{CH}_{2}$ groups either because of their chemical composition or because of their packing density (See Fig. 10.) [77]. More quan titative data analysis is possible if deuteration is used in addition. In this way, the content of [D62] DPPC in coexisting domains of DOPC/d62 DPPC bilayers was quantitatively measured as a func tion of cholesterol content in Ref. [78].

\subsection{Cellular systems}

Membrane model systems of the types described above have the advantage that they consist of only a few different molecular species often only one or two with known vibrational spectra and well defined concentrations. Compared to cellular systems, which contain a large number of species with different spectra in unknown concentrations, this makes the spectral interpretation much simpler. However, potential applications in live cell and live animal imaging have been the most important motivation for the development of non linear vibrational microscopy techniques. Apart from difficulties due to the complex nature of real world bio logical samples, live cell and animal investigations are faced with the additional problem that the excitation light intensities should be kept at a level which does not induce any photodamage. For all the techniques discussed in this review, the situation is compa rable to that encountered in two photon excited fluorescence microscopy. Since all approaches employ pulsed lasers excitation in order to induce non linear optical effects in the samples, there are two damage regimes which deserve attention. In a linear re gime, i.e. when considering effects caused by linear absorption of laser light by the sample, mainly local heating is the cause of the damage. In order to reduce this problem, the excitation wave lengths used are usually chosen to lie in the near infrared spectral region, where the absorption of most cells and tissues is rather weak. Use of intense pulsed lasers, however, can also lead to unde sired non linear effects which might e.g. result in multi photon excitation or avalanche breakdown and the ionization of molecules in the microscope focus. Several studies have addressed the prob lem of phototoxicity of pulsed laser excitation [79 81]. While these give some insight into the parameters relevant for the cause of photodamage, the complexity of the damage process compli cates any prediction of the amount of photodamage. This is due to the broad range of important variables such as the exact nature of the biological sample, the treatment of the cells (temperature, culture medium, atmosphere), the laser pulse duration, the pulse energy, the excitation wavelength, and the laser repetition rate, to name only the most important ones. A general issue which has to be mentioned in this respect is that the term photodamage is ill defined. Often biological samples can cope with seemingly dramatic photodamage such as membrane blebbing, commonly re garded as a clear sign of photodamage [81]. Earlier blebbing will, for example, not necessarily inhibit cell division. On the other hand, careful inspection of the samples often shows laser induced modifications such as enhanced autofluorescence even if no other apparent sign of damage is present. Thus, care has to be taken to avoid laser excitation related data artifacts.

For the proper interpretation of cellular images obtained with non linear vibrational microscopy, it is necessary to recall the sig nal generation mechanisms and imaging schemes described in 3. In nearly all experiments, the signal will be generated in a focal spot of the size of $1 \mu \mathrm{m}^{3}$. This means that a concentration of $1 \mu \mathrm{mol}$ 


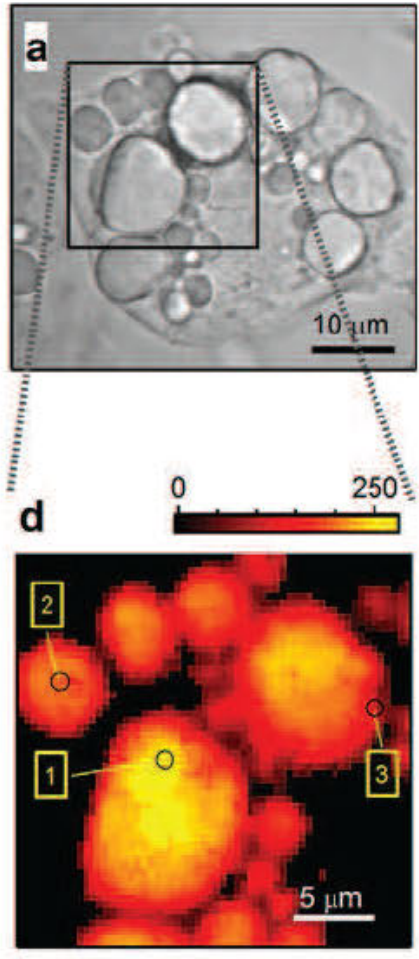

Integrated intensity
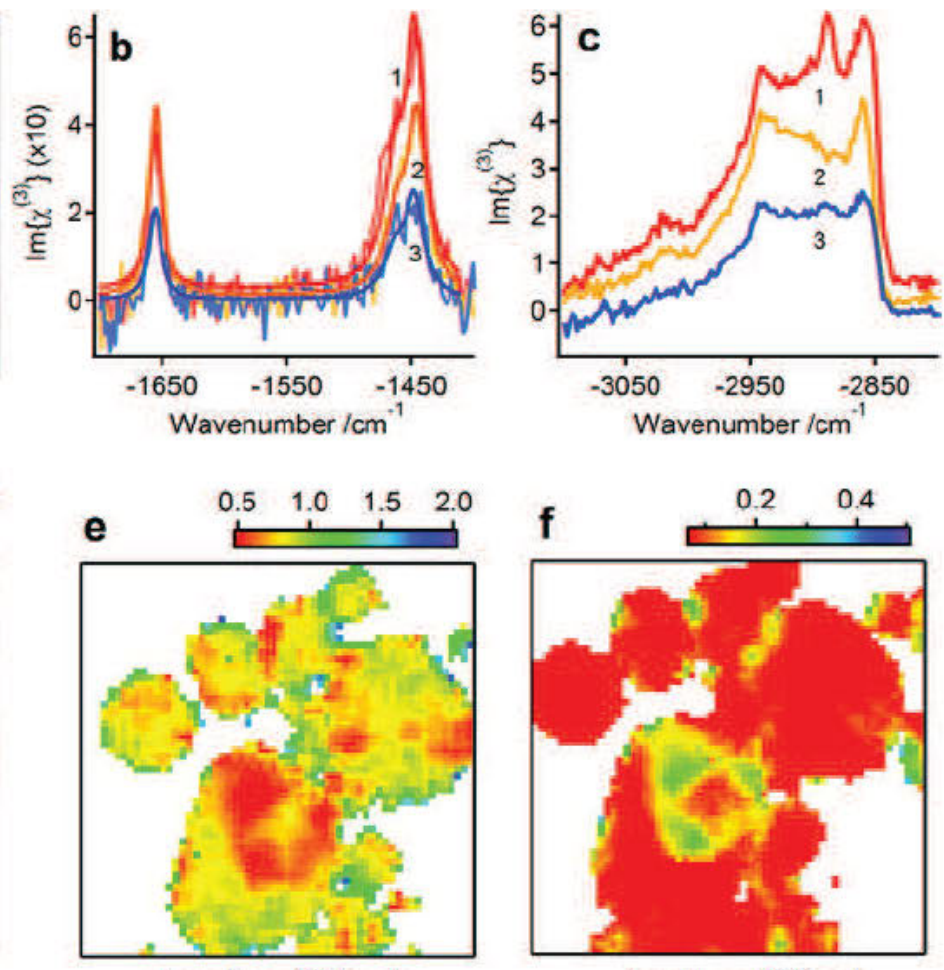

$\mathrm{I}_{-1650} \mathrm{I}_{-1450}\left(\mathrm{CC}_{\text {unsat }}\right)$

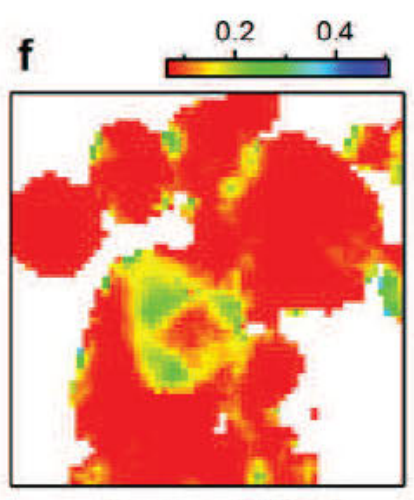

$\mathrm{I}_{-2880} \mathrm{I}_{-2845}\left(\mathrm{CC}_{\mathrm{crder}}\right)$

Fig. 11. Multiplex CARS spectral imaging of lipid droplets in fixed 3T3-L1 cells. (a) Bright-field image of an adipocyte incubated with a 1:3 mixture (mol/mol) of linolenic and palmitic acid. Marked region was imaged with multiplex CARS microscopy. Images are derived with contrast based on (d) lipid concentration (integrated intensity), (e) C = C concentration derived from CC-stretch region $\left(I_{1650} / I_{1450}\right)$, and (f) acyl-chain order $\left(I_{2880} / I_{2845}\right)$. (b and c) Representative Im (Chi $\left.{ }^{(3)}\right)$ spectra were recorded at locations indicated (in d) for CC-stretch and CH-stretch spectral regions, respectively. A least-squares fit of a sum of three Lorentzians to the data is also shown (b). Acquisition time was $20 \mathrm{~ms}$ for a multiplex CARS spectrum at every pixel position ( 1 min/image). Adapted from Ref. [86].

corresponds to roughly $10^{3}$ molecules per excitation volume. Typ ical sensitivity limits of CARS as well as of SRS, however, are in the range of $10^{5} 10^{6}$ oscillators. As a result, CRS imaging techniques can detect small sample structures down to sizes of a few hundred $\mathrm{nm}$ in diameter, as long as the local concentrations of molecules is high. These conditions are met e.g. in cellular vesicles. CRS instead is less suited for monitoring small variations in evenly distributed diluted compounds. For this reason and due to the mentioned strong CRS signal of lipids, non linear vibrational microscopy has extensively been used to study lipid droplets (LD). During the last two decades, it has become apparent that LDs are cellular organ elles with a well defined internal structure. A monolayer of phos pholipds forms their outer membrane, into which a broad variety of proteins are inserted [82]. The interior of the LD contains neutral lipids (mainly triglycerides and sterol esters). LDs participate in a broad variety of physiological processes and are found in many dif ferent cell types. Their sizes vary from some tens of $\mathrm{nm}$ to tens of $\mu \mathrm{m}$ in diameter in differentiated adipocytes. Since they have an ac tive role in fat metabolism, LDs are dynamic organelles both con cerning their intracellular location as well as their size. CARS microscopy has been used to study the movement of LDs in live cells. These tracking experiments, which can be performed at vi deo rate image acquisition times, reveal that LDs are actively transported in cells. This transport is mediated by microtubule re lated motor proteins $[83,84]$ and is commonly bidirectional, i.e. it can be directed towards the plus or the minus end of the micro tubules, which can be explained by the simultaneous presence of different motor proteins on the LDs. The balance between the two transport directions can be changed e.g. after infection with the hepatitis C virus [85]. Multiplex CARS microscopy has been used to monitor the fat composition of LDs in HeLa cells and adipocytes under different dietary conditions [86,87]. In these works, ratios between intensities observed for different vibrational bands were used to infer information about the acyl chain order and the ratio between saturated and unsaturated fatty acids. While some of these markers provided the expected results, it was also seen that the prominent resonance of unsaturated fatty acids at $3020 \mathrm{~cm}{ }^{1}$ failed to provide quantitative information about the lo cal concentration. Nevertheless, use of this approach allowed the identification of variations in the composition of LDs in individual cells. (See Fig. 11.)

A major application of CRS microscopy in quantitative studies of LDs has been the monitoring of LD numbers and sizes. As men tioned before, CRS microscopy is suited for LD sizes down to diam eters of approximately $100 \mathrm{~nm}$. Small LDs are mainly observed in cells not specialized for fat storage, but are also present in adipo cytes held under lipolytic conditions. CARS microscopy has suc cessfully been used to quantitatively monitor the size of LDs in yeast cells [88]. The morphological dynamics of LDs are most strik ing in adipocytes which can be derived from mesenchymal stem cells. CARS microscopic inspection of these stem cells shows only few LDs of very small size. Differentiation into adipocytes leads to a drastic change in LD number and size over approximately two weeks. Shortly after the induction of differentiation, numerous small LDs are formed which fuse to finally form one unilocular li pid droplet per cell with diameters exceeding $10 \mu \mathrm{m}$. This process has been followed using CARS microscopy (Fig. 12). The specific virtue of CARS microscopy here is the possibility to perform long term experiments over the course of more than a week on the same sample without the occurrence of marker bleaching. Analysis of the data gives detailed insight into the fusion behavior of LDs [89]. However, large LDs are not only found in adipocytes. 

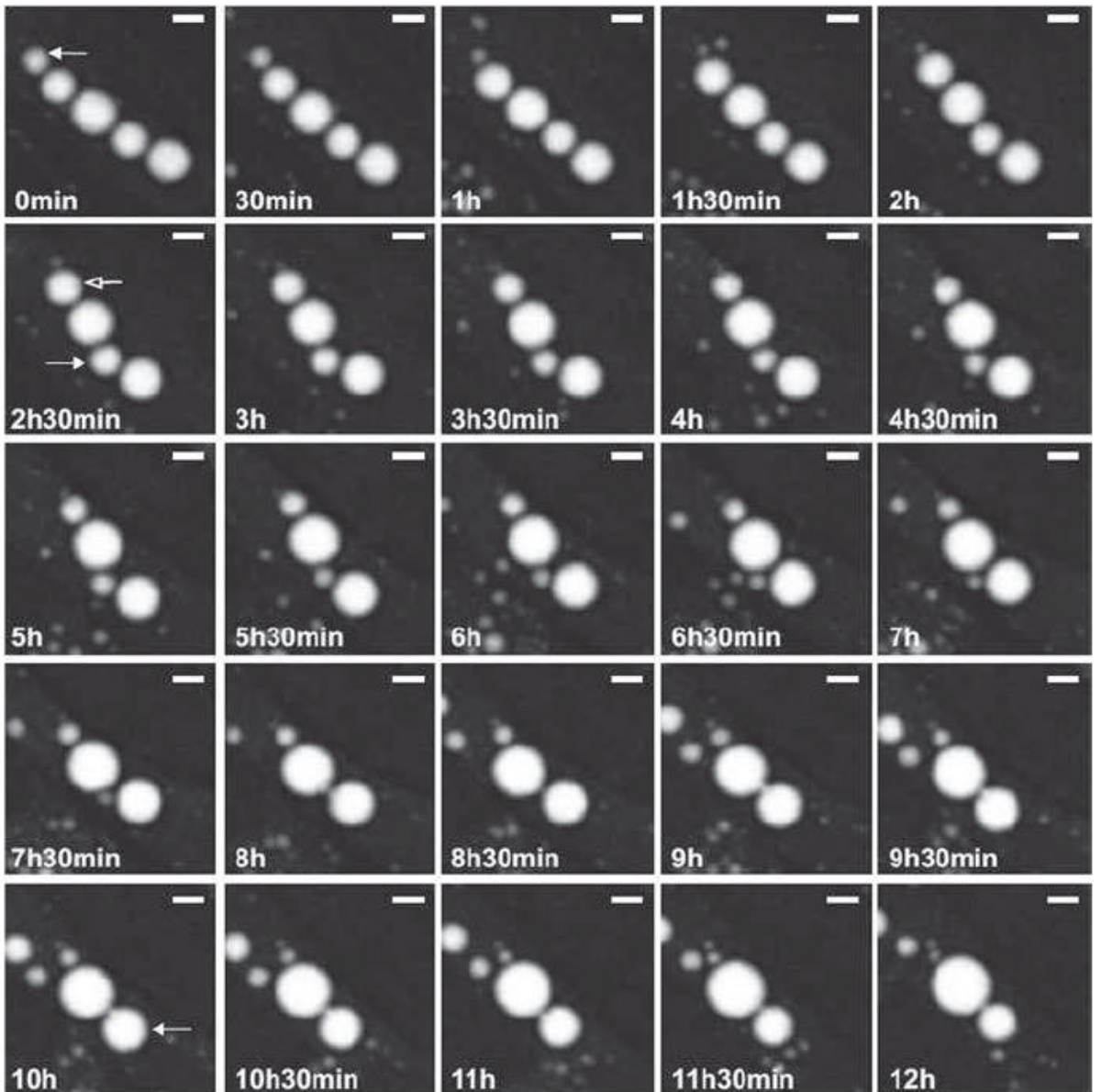

Fig. 12. CARS imaging of LD growth in human adipose-derived stem cells. Lipid acceptors can turn into lipid donors: A lipid donor (solid arrow, first panel) is almost completely absorbed over time by a closely associated lipid acceptor below. Later on, this lipid acceptor in turn serves as a lipid donor (open arrow, second panel) for a LD, which simultaneously absorbs a second LD (solid arrow, second panel). Finally, this LD starts to absorb a third LD (solid arrow, last panel). The completion of one LD fusion takes more than $2 \mathrm{~h}$. During the fusion process, one contact point is maintained. CARS images were acquired at $2845 \mathrm{~cm}{ }^{1}$. Maximum-intensity projections of $3 \mathrm{D}$ stacks acquired at the indicated time points. Bar $=10 \mu \mathrm{m}$. Adapted from [89].

(a)<smiles>O=C(Nc1ccccc1)c1cc([N+](=O)[O-])ccc1Cl</smiles>

(b)

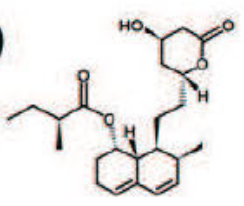

(c)

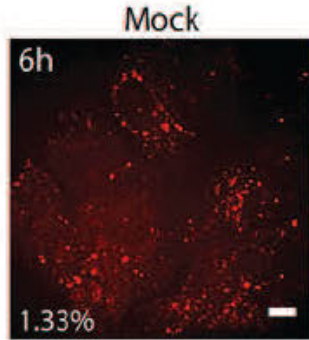

+ Benzamide

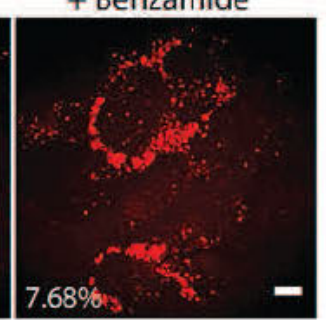

(d)
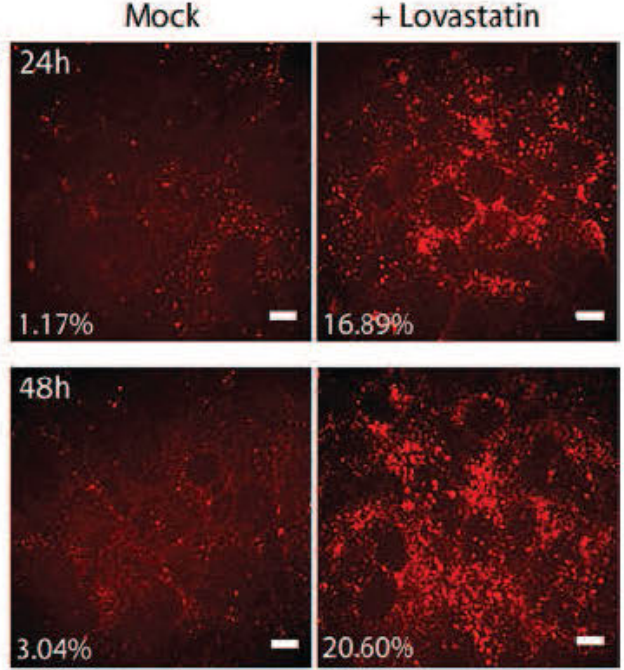

Fig. 13. CARS images showing changes in lipid distributions in Huh-7.5 cells after treatment with the small molecules BA and lovastatin. (a) Structure of the PPAR $\alpha$ (peroxisome proliferator activated receptor $\alpha$ ) antagonist 2-chloro-5-nitro-N-(pyridyl) benzamide (BA) and (b) structure of the HMG (3-hydroxy-3-methyglutaryl)-CoA reductase inhibitor lovastatin. (c) Images depicting the effects of $75 \mu \mathrm{M}$ BA on Huh-7.5 cells incubated in culture for $6 \mathrm{~h}$. (d) Images showing the effects of $50 \mu \mathrm{M}$ lovastatin treated Huh-7.5 cells for either 24 or $48 \mathrm{~h}$. The values on the bottom left corner of the CARS images represent the results of a voxel analysis indicating the lipid droplet volume per cell (average of 5 cells per sample). Scale bar: $10 \mu \mathrm{m}$. Adapted from [95]. 
CARS microscopy has revealed that also enterocytes, absorptive cells of the small intestine, contain pools of LDs which can grow to sizes of nearly $10 \mu \mathrm{m}$ in diameter [90]. The pool of these LDs is dynamic and the enzymatic regulation of these dynamics has been investigated using CARS microscopy in Ref. [91,92].

CRS microscopy has also been used to monitor pathological changes in LD structures and dynamics. Changes in the lipid metabolism are induced by various different diseases. One exam ple are infections by the hepatitis C virus (HCV) which are known to affect the lipid metabolism of the host [93] and which result in changes in the cellular accumulation of LDs. CARS microscopy has been used to show that HCV replication can be suppressed by the action of a small molecule inhibitor. Application of the inhibitor to infected HUH 7 cell cultures thereby leads to a clearly detectable increase of cellular lipid accumulation [94] and a disruption of HCV replication. A combination of two photon excited fluores cence experiments and CARS microscopy shows that the latter ef fect is caused by dispersion of the HCV RNA (Fig. 13) [95].

CRS microscopy has also proven to be a valuable tool for study ing lipid related mechanisms of cancer metastasis. While it has been noticed that lipid rich tumors exhibit a stronger tendency to metastasis, the details of this process have not been understood. Application of CARS microscopy in vitro and in vivo has shown that excess free fatty acids lead to a phase separation in the cell mem branes disrupting cell cell contacts resulting in a higher cell motil ity [96]. In these experiments, CARS based intravital flow cytometry was used. For this purpose, a superficial vein in a mouse ear was monitored using CARS microscopy. Simultaneous two photon excited fluorescence imaging then served for the iden tification of tumor cells. This example shows that the fast imaging capabilities of CARS microscopy lend themselves also to the application in analytical techniques such as cytometry, possibly complementing existing fluorescence based cytometry approaches $[97,98]$.

\subsection{Tissues and model organisms}

As a label free multiphoton microscopy technique with intrin sic optical sectioning (3D resolution) and substantial penetration depth, CRS offers unique opportunities to image tissues and model organisms. The first application of CRS to live imaging was shown in Ref. [52] where the skin tissue of a mouse ear was imaged at video rate using CARS in a backscattering geometry, namely for ward CARS which was scattered in the tissue and collected in epi direction. A similar demonstration with SRS was subsequently reported in Ref. [44,99]. These works showed the ability to distin guish sebaceous glands, corneocytes and adipocytes label free and to follow dynamic processes such as the diffusion of mineral oils and drugs into the skin. The potential of CARS combined with two photon autofluorescence for 'optical skin biopsy' in vivo and in turn diagnosis of skin cancer in human patients was shown in Ref, [100].

Live animal imaging with CRS has also been shown on the mod el organism C. elegans specifically addressing lipid metabolism. Owing to its small size, optical transparency, its ability to support lipid droplet expansion to $>10 \mu \mathrm{m}$ size and its extensively studied genome, C. elegans has become an attractive model for studying fat storage in vivo combining sub cellular resolution with whole ani mal behavior. CARS microscopy was used to quantify changes in li pid storages in genetic mutants [101 103] and the effect of cholesterol lowering drugs [104]. Noticeable findings included the visualization of hypodermal lipid droplets that were not visible or quantifiable by fluorescence using Nile red, thus highlighting the advantage of label free microscopy [101] (see also Fig. 14A). Non linear optical imaging has also been used to study embryo genesis in model organisms. Here, by following the lipid droplet distribution the different stages of development can be visualized, as has first been shown in zebrafish embryos using THG micros copy. Recently, early stage development based on lipid droplet tracking has also been studied in Drosophila embryos using femto second SRL microscopy [105] (see also Fig. 14B).

Nerve tissues have also been widely investigated with CRS, since the myelin sheath that coats nerve axons is lipid rich and thus provides strong CRS contrast. Demyelination is a hallmark for many neurodegenerative diseases. Real time imaging of intact nerve tissues by CRS therefore offers a unique opportunity to elu cidate the cellular mechanism of demyelination disorders. Wang et al. [109] reported the first application of CARS to visualize myelin sheets in explanted spinal cord tissues of guinea pigs. Following studies included the characterization in real time of
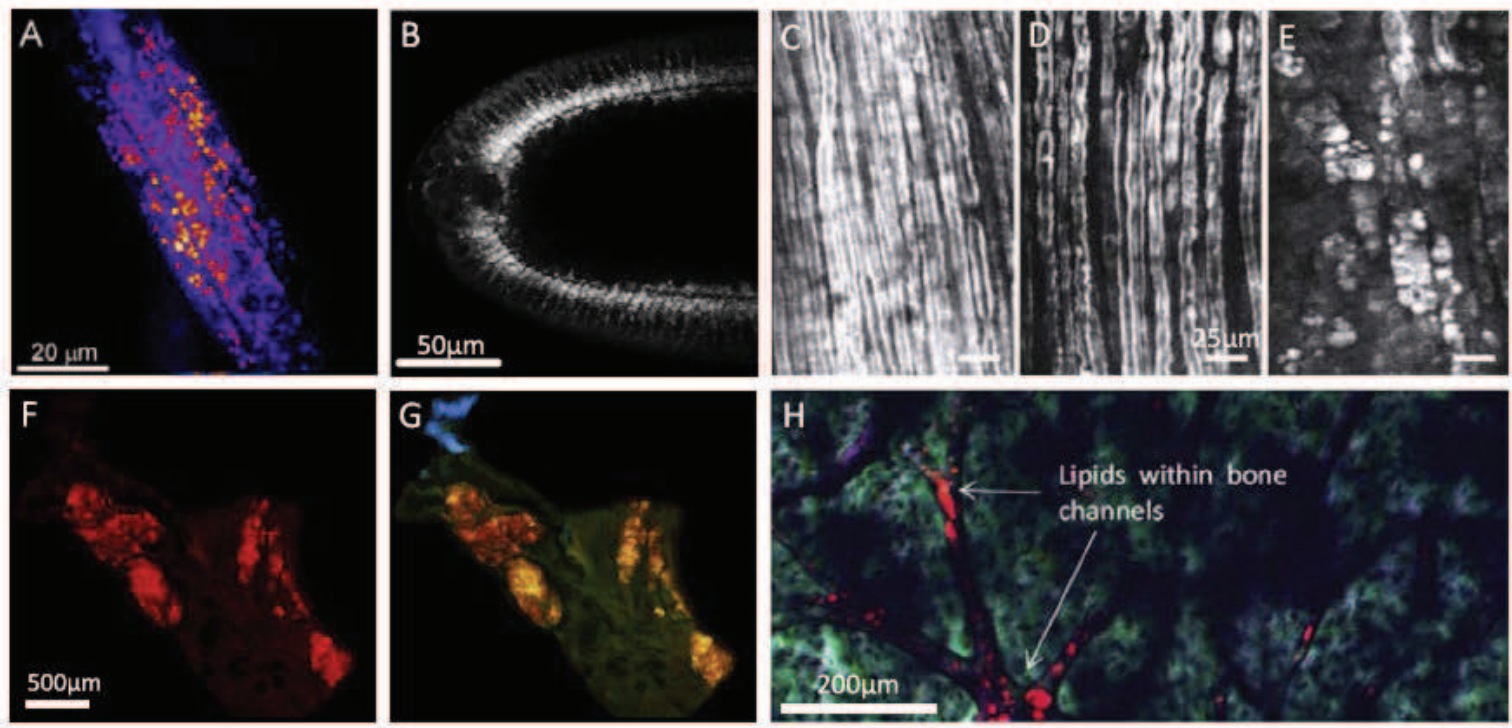

Fig. 14. (A) CARS microscopy of lipid droplets in C. elegans (adapted from Ref. [101]). (B) SRL image of lipid droplets in a Drosophila embryo (adapted from Ref. [105]). (C-E) CARS of a crushed rat sciatic nerve ranging from the healthy (C), proximal (D) and distal (E) region of the lesion (adapted from Ref. [106]). (F, G) CARS and multimodal SHC (blue)/TPF (green)/CARS (red) images of the entire descending aorta of an ApoE-deficient mouse fed a Western diet (adapted from Ref. [107I). H) SRS multicolor image of an equine articular cartilage (Blue: carbonate resonance at $1070 \mathrm{~cm}{ }^{2}$. Green: Phosphate resonance at $959 \mathrm{~cm}$ '. Red: Lipid resonance at $2840 \mathrm{~cm}{ }^{1}$ (adapted from Ref. [108]). 
lysophosphatidylcholine induced myelin degradation ex vivo and in vivo in mice [110], the study of the microenvironment of the sci atic nerve in a living mouse by CARS and SHG [111], the ability of block copolymer micelles to repair spinal cord injuries [112], the response of myelin to high frequency electrical stimulation for therapeutic purposes [113], and the real time in vivo monitoring of demyelination and subsequent remyelination of a rat sciatic nerve after controlled injury [106] (see also Fig. 14C E). In experi ments of this type, the spectroscopic information of CARS micros copy can be complemented with other non linear optical responses of the sample, which can be read out simultaneously. Thus, different aspects related to the healing of spinal cord injuries has been followed by simultaneous monitoring of two photon excited autofluorescence from macrophages (inflammatory re sponse), of SHG indicative for the formation of collagen fibers (scar formation), and of CARS depicting nervous tissue [114].

The application of CRS to brain imaging has also been investi gated. Evans et al. [115] demonstrated the use of CARS microscopy in identifying normal brain structures and primary glioma in fresh unfixed and unstained ex vivo mouse brain tissue. More recently, chemoselective imaging of fresh mouse brain tissue via multiplex CARS microscopy [116], and multimodal CARS, SHG, and TPF on unstained tissue sections from pig brain have been demonstrated [117]. Furthermore, a novel combination of SRS and two photon photothermal lensing was recently shown to provide simultaneous visualisation of cortical microvasular morphology and surrounding cellular structures in mice brain slices [118].

Another major clinically relevant application of CRS is the study of lipids in atherosclerotic plaques. Existing diagnostic tools for atherosclerosis such as magnetic resonance imaging and ultra sound tend to detect only advanced calcified plaques and are inad equate for early diagnosis. In addition the diagnosis of vulnerable plaques require a chemical evaluation of their composition which is lacking in these techniques. CRS imaging overcomes these limi tations as shown in Ref. [119] where multiplex CARS has been used to identify the chemical profile of four types of atherosclerotic lip ids in mice aortas ex vivo. Multimodal CARS/SHG/TPF was used in Ref. [120,121] to visualize the composition of arterial walls and atherosclerotic plaques. The impact of a high fat high cholesterol diet on the composition of atherosclerotic plaques in apolipopro tein E deficient mouse model was reported in Ref. [107] using mul timodal CARS/SHG/TPF (see also Fig. $14 \mathrm{~F}$ and G), and the identification of cholesterol crystals in plaques of atherosclerotic mice using multiplex CARS imaging was shown in Ref. [122]. Fur thermore, multimodal CARS/SHG/TPF was used to characterize atherosclerotic plaque development in myocardial infarction prone rabbits [123] and stent induced coronary artery stenosis in pigs [124].

Other applications of CRS to tissues included imaging of liver and intestine. Specific imaging of fat droplets in intact liver tissues was performed by CARS microscopy in Ref. [125] and used to quan tify the hepatic fat content through image analysis while eliminat ing laborious procedures required by traditional histopathological examination. Multimodal CARS/SHG/TPF was shown to be a valu able tool to assess liver steatosis and fibrosis in rats [126]. Ex vivo and in vivo CARS imaging of small intestine of mice during dietary fat absorption was shown in Ref. [90,92].

Also to be noted are CRS imaging of breast tissue and lung tis sue, for the purpose of cancer diagnosis. Specifically, a knowl edge based CARS microscopy system was developed in Ref. [127] for label free imaging, pattern recognition, and classification of cells and tissue structures from human patients in order to differ entiate lung cancer from non neoplastic lung tissues and identify lung cancer subtypes. The differential diagnosis of breast cancer using CARS in human breast tissues was reported in Ref. [128]. The use of combined confocal Raman and high speed CARS imaging for label free determination of apical polarity of human live mammary acini in 3D culture was shown in Ref. [129] to be an effective method for the rapid identification of risk factors that initiate the very early stage of epithelial neoplasia. Moreover, in Ref. [130] CARS was used to validate an adipose tumor epithelial cell co culture system designed to reproduce the in vivo mammary environment.

Within the remit of tissue engineering, multimodal CARS/SHG has been used recently to visualize the $3 \mathrm{D}$ cell arrangement in microporous bacterial cellulose scaffolds seeded with osteoprogen itor cells [131], to study the interaction between rat marrow stro mal cells and engineered extracellular matrices [132], and to characterize bovine smooth muscle cells and their proliferation, migration, and differentiation in hydrogel cellulose scaffolds [133]. SRS has also been applied to unstained samples of articular cartilage enabling the investigation of living cells within fresh tis sue and the identification of variations in protein and lipid content within the cells, pericellular matrix and interterritorial matrix [108] (see also Fig. 14H).

Finally, an upcoming area of biomedical interest is the study of nanoparticle uptake in cells and tissues. The motivation for these studies is either an interest in the toxicity of nanoparticles or in their pharmaceutical application. While CARS microscopy has been applied in studies of nanoparticle toxicity [134], much more work is currently undertaken to study drug delivery using non linear vibrational microscopy. Examples include Ref. [135] where CARS microscopy was used to image peptide drug nanofibers delivered across the blood brain barrier in mice brain tissues ex vivo. A com bination of CARS, SHG and TPF microscopy was used in Ref. [136] as a multimodal label free method for pinpointing orally adminis trated polymeric nanoparticles within the stomach, intestine, gall bladder and liver in mice tissue sections.

\section{Conclusions and outlook}

In conclusion, we have shown that CRS microscopy is a versatile new tool for studying a variety of different samples. With its spe cific strength of allowing video rate imaging of unlabeled samples while providing chemically specific contrast, it complements exist ing linear and non linear optical microscopy techniques. After more than a decade of technological development of different CRS approaches, the techniques are now mature and can be applied also by non specialists. This is witnessed by the fact that the majority of publications in the field are now focused on biological applications of CRS microscopy, and commercial CRS microscopes are now available. Specifically, we pointed out many applications relevant to lipid biology due to the strong CRS signals of lipids. This will most likely continue to be the case, with bio medical applica tions receiving even more attention in the future.

\section{Acknowledgements}

AZ gratefully acknowledges financial support by the German Federal Ministry of Education and Research in the framework of the MICROQUANT project. PB acknowledges the UK EPSRC Re search Council for her Leadership fellowship award (Grant no. $\mathrm{EP} / \mathrm{I005072/1)}$. PB and WL are funded by the UK BBSRC Research Council (Grant no. BB/H006575/1) and the UK EPSRC Research Council (Grant no. EP/H45848/1).

\section{References}

[1] Denk W, Strickler J, Webb W. Two-photon laser scanning microscopy. Science 1990;248:73-6.

[2] Fukumoto S, Fujimoto T. Deformation of lipid droplets in fixed samples. Histochem Cell Biol 2002;118:423-8. 
[3] Nan X, Cheng JX, Xie XS. Vibrational imaging of lipid droplets in live fibroblast cells with coherent anti-stokes raman scattering microscopy. J Lipid Res 2003;44(11):2202. 8.

[4] Ohsaki Y, Shinohara Y, Suzuki M, Fujimoto T. A pitfall in using bodipy dyes to label lipid droplets for fluorescence microscopy. Histochem Cell Biol 2010;133(4):477. 80.

[5] Brown E, McKee T, diTomaso E, Pluen A, Seed B, Boucher Y, et al. Dynamic imaging of collagen and its modulation in tumors in vivo using secondharmonic generation. Nat Med 2003;9(6):796-800.

[6] Pfeffer CP, Olsen BR, Ganikhanov F, Legare F. Multimodal nonlinear optica imaging of collagen arrays. J Struct Biol 2008;164(1):140-5.

[7] Barad Y, Eisenberg H, Horowitz M, Silberberg Y. Nonlinear scanning laser microscopy by third harmonic generation. Appl Phys Lett 1997;70:922-4.

[8] Squier J, Müller M, Brakenhoff G. Third harmonic generation microscopy. Opt Express 1998;3:315-24.

[9] Débarre D, Supatto W, Pena A-M, Fabre A, Tordjmann T, Combettes L Schanne-Klein M-C, Beaurepaire E. Imaging lipid bodies in cells and tissues using third-harmonic generation microscopy. Nat Methods 2006;3:47-53.

[10] Débarre D, Beaurepaire E. Quantitative characterization of biological liquids for third-harmonic generation microscopy. Biophys J 2007:92(2):603-12.

[11] Olivier N, Luengo-Oroz MA, Duloquin L, Faure E, Savy T, Veilleux I, Solinas X, Debarre D, Bourgine P, Santos A, Peyrieras N, Beaurepaire E. Cell lineage reconstruction of early zebrafish embryos using label-free nonlinear microscopy. Science 2010;329(5994):967. 71

[12] Farrar MJ, Wise FW, Fetcho JR, Schaffer CB. In vivo imaging of myelin in the vertebrate central nervous system using third harmonic generation microscopy. Biophys J 2011;100(5):1362-71.

[13] Duncan M, Reintjes J, Manuccia T. Scanning coherent anti-stokes raman microscope. Opt Lett 1982;7:350-2.

[14] Zumbusch A, Holtom G, Xie X. Three-dimensional vibrational imaging by coherent anti-stokes raman scattering. Phys Rev Lett 1999;82:4142-5.

[15] Ploetz E, Laimgruber S, Berner S, Zinth W, Gilch P. Femtosecond stimulated raman microscopy. Appl Phys B-Lasers Opt 2007;87(3):389-93.

[16] Freudiger CW, Min W, Saar BG, Lu S, Holtom GR, He CW, Tsai JC, Kang JX, Xie XS. Label-free biomedical imaging with high sensitivity by stimulated raman scattering microscopy. Science 2008;322(5909):1857-61.

[17] Nandakumar P, Kovalev A, Volkmer A. Vibrational imaging based on stimulated raman scattering microscopy. New J Phys 2009;11(3):033026.

[18] Long DA. The Raman effect: a unified treatment of the theory of Raman scattering by molecules. Wiley; 2002

[19] Sigma aldrich product catalogue 2012. <http://www.sigmaaldrich.com catalog/product/sigma/c8667>.

[20] Langbein W, Borri P. Resonant nonlinear optical microscopy. In: Proceedings of the Varenna School Course CLXXXI Microscopy. Biophotonics, in press.

[21] Vartiainen E, Rinia HA, Müller M, Bonn M. Direct extraction of raman lineshapes from congested cars spectra. Opt Express 2006;14:3622-30.

[22] Rinia HA, Bonn M, Müller M, Vartiainen EM. Quantitative cars spectroscopy using the maximum entropy method: The main lipid phase transition. Chen Phys Chem 2007:8(2):279-87.

[23] Peterson CW, Knight BW. Causality calculations in the time domain: An efficient alternative to the Kramers-Kronig method. J Opt Soc Am 1973;63(10):1238-42. http://dx.doi.org/10.1364/JOSA.63.001238.

[24] Liu Y, Lee YJ, Cicerone MT. Broadband cars spectral phase retrieval using a time-domain Kramers-Kronig transform. Opt Lett 2009:34:1363.

[25] Ganikhanov F, Evans C, Saar B, Xie X. High-sensitivity vibrational imaging with frequency modulation coherent anti-stokes raman scattering (FM cars) microscopy. Opt Lett 2006;31:1872-4.

[26] Burkacky O, Zumbusch A, Brackmann C, Enejder A. Dual-pump coherent antistokes raman scattering microscopy. Opt Lett 2006;31:3656-8.

[27] Rocha-Mendoza I, Langbein W, Watson P, Borri P. Differential coherent antistokes raman scattering microscopy with linearly chirped femtosecond laser pulses. Opt Lett 2009;34(15):2258-60.

[28] Langbein W, Rocha-Mendoza I, Borri P. Coherent anti-stokes raman microspectroscopy using spectral focusing: theory and experiment. I Raman Spectrosc 2009;40(7):800-8

[29] Di Napoli C, Masia F, Pope I, Otto C, Langbein W, Borri P. Chemically-specific dual/differential cars micro-spectroscopy of saturated and unsaturated lipid droplets. J Biophotonics, in press. http://dx.doi.org/10.1002/jbio.201200197.

[30] Rocha-Mendoza I, Borri P, Langbein W. Quadruplex cars micro-spectroscopy. Raman Spectrosc 2013;44:255

[31] Cheng J-X, Book LD, Xie X. Polarization coherent anti-stokes raman scattering microscopy. Opt Lett 2001;105:1341-3.

[32] Cheng J-X, Volkmer A, Xie X. Theoretical and experimental characterization of coherent anti-stokes raman scattering microscopy. J Opt Soc Am B 2002; 19:1363-75

[33] Lu F, Zheng W, Huang Z. Elliptically polarized coherent anti-stokes raman scattering microscopy. Opt Lett 2008;33:2842.

[34] Heinrich C, Bernet S, Ritsch-Marte M. Wide-field coherent anti-stokes raman scattering microscopy. Appl Phys Lett 2004;84:816-8.

[35] Heinrich C, Hofer A, Ritsch A, Ciardi C, Bernet S, Ritsch-Marte M. Selective imaging of saturated and unsaturated lipids by wide-field cars-microscopy. Opt Express 2008:16(4):2699-708.

[36] Toytman I, Simanovskii D, Palanker D. On illumination schemes for wide-field cars microscopy. Opt Express 2009;17:7339.
[37] Beversluis MR, Stranicka SJ. Enhanced contrast coherent anti-stokes raman scattering microscopy using annular phase masks. Appl Phys Lett 2008:93:231115.

[38] Potma E, Evans C, Xie X. Heterodyne coherent anti-stokes raman scattering cars) imaging. Opt Lett 2006;31:241-3.

[39] Jurna M, Korterik JP, Otto C, Herek JL, Offerhaus HL. heterodyne detection, Vibrational phase contrast microscopy by use of coherent anti-stokes raman scattering. Phys Rev Lett 2009;103:043905. http://dx doi org/101103/ PhysRevLett 103043905

[40] Jones G, Marks D, Vinegoni C, Boppart S. High-spectral resolution coherent anti-stokes raman scattering with interferometrically detected broadband chirped pulses. Opt Lett 2006;31:1543-5.

[41] Lee ES, Lee JY. Coherent anti-stokes raman scattering spectral interferometry with large spectral phase gradient induced by highly dispersive optical elements. Opt Lett 2008;33(24):3016-8. http://dx.doi.org/10.1364/ OL.33.003016.

[42] Pope I, Langbein W, Watson P, Borri P. Simultaneous hyperspectral differential-cars, tpf and shg microscopy with a single 5 fs ti:sa laser. Opt Express 2013;21(5):7096-106.

[43] Ozeki Y, Dake F, Kajiyama S, Fukui K, Itoh K. Analysis and experimental assessment of the sensitivity of stimulated raman scattering microscopy. Opt Express 2009;17(5):3651-8. http://dx.doi.org/10.1364/OE.17.003651.

[44] Saar BG, Freudiger CW, Reichman J, Stanley CM, Holtom GR, Xie XS. Videorate molecular imaging in vivo with stimulated raman scattering. Science 2010;330(6009):1368-70.

[45] Fu D, Lu FK, Zhang X, Freudiger C, Pernik DR, Holtom G, Xie XS. Quantitative chemical imaging with multiplex stimulated raman scattering microscopy. J Amer Chem Soc 2012;134(8):3623-6 (iSI Document Delivery No.: 903ZE Times Cited: 0 Cited Reference Count: 23 Fu, Dan Lu, Fa-Ke Zhang, Xu Freudiger, Christian Pernik, Douglas R. Holtom, Gary Xie, Xiaoliang Sunney Nih t-r01[ir01eb010244-01] We thank Dr. Xiaohui Ni for providing the mouse skin sample. This work was supported by the NIH T-R01 (IR01EB010244-01) Award to X.S.X. Amer chemical soc, Washington.).

[46] Freudiger CW, Min W, Holtom GR, Xu BW, Dantus M, Xie XS. Highly specific abel-free molecular imaging with spectrally tailored excitation-stimulated raman scattering (ste-srs) microscopy. Nat Photonics 2011;5(2):103-9.

[47] Ploetz E, Marx B, Klein T, Huber R, Gilch P. A $75 \mathrm{mhz}$ light source for femtosecond stimulated raman microscopy. Opt Express 2009;17(21):18612-20. http://dx doi org/10 1364/OE 17018612

[48] Kong L, Ji M, Holtom GR, Fu D, Freudiger CW, Xie XS. Multicolor stimulated raman scattering microscopy with a rapidly tunable optical parametric oscillator. Opt Lett 2013;38(2):145-7. http://dx doi org/10 1364/ OL.38.000145.

[49] Ozeki Y, Umemura W, Otsuka Y, Satoh S, Hashimoto H, Sumimura K, Nishizawa N, Fukui K, Itoh K. High-speed molecular spectral imaging of tissue with stimulated Raman scattering. Nat Photonics 2012;6(12):844-50. http:/ dx.doi.org/10.1038/NPHOTON.2012.263.

[50] Malinovskaya SA, Malinovsky VS. Chirped-pulse adiabatic control in coherent anti-stokes raman scattering for imaging of biological structure and dynamics. Opt Lett 2007;32(6):707-9. http://dx.doi.org/10.1364/ OL.32.000707.

[51] Boyd RW. Nonlinear optics. Elsevier; 2003.

[52] Evans C, Potma E. M.P. haag, D. Côté, C. Lin, X. Xie, Chemical imaging of tissue in vivo with video-rate coherent anti-stokes raman scattering microscopy. Proc Natl Acad Sci USA 2005;102:16807-12.

[53] Cheng J-X, Volkmer A, Book LD, Xie X. An epi-detected coherent anti-stokes raman scattering (e-cars) microscope with high spectral resolution and high sensitivity. J Phys Chem B 2001;105:1277-80.

[54] Cheng J-X, Xie X. Coherent anti-stokes raman scattering microscopy: Instrumentation, theory, and applications. J Phys Chem B 2004;108:827-40.

[55] Evans CL, Xie XS. Coherent anti-stokes raman scattering microscopy: Chemical imaging for biology and medicine. Ann Rev Anal Chem 2008;1:883-909.

[56] Hellerer T, Enejder A, Zumbusch A. Spectral focusing: High spectral resolution spectroscopy with broad-bandwidth laser pulses. Appl Phys Lett 2004;85:25-7.

[57] Pegoraro AF, Ridsdale A, Moffatt DJ, Jia Y, Pezacki JP, Stolow A. Optimally chirped multimodal cars microscopy based on a single ti:sapphire oscillator. Opt Express 2009;17:2984-96.

[58] Langbein W, Rocha-Mendoza I, Borri P. Single source coherent anti-stokes raman microspectroscopy using spectral focusing. Appl Phys Let 2009;95(8):081109.

[59] von Vacano B, Motzkus M. Time-resolving molecular vibration for microanalytics: single laser beam nonlinear raman spectroscopy in simulation and experiment. Phys Chem Chem Phys 2008;10:609-756.

[60] Dudovich N, Oron D, Silberberg Y. Single-pulse coherently controlled nonlinear raman spectroscopy and microscopy. Nature 2002;418:512-4.

[61] Cheng JX, Volkmer A, Book LD, Xie XS. Multiplex coherent anti-stokes raman scattering microspectroscopy and study of lipid vesicles. J Phys Chem B 2002;106(34):8493-8.

[62] Müller M, Zumbusch A. Coherent anti-stokes raman scattering microscopy. Chem Phys Chem 2007:8(15):2156-70.

[63] Kano H. H. o Hamaguchi, Ultrabroadband (2500 cm[sup -1]) multiplex coherent anti-stokes raman scattering microspectroscopy using a 
supercontinuum generated from a photonic crystal fiber. Appl Phys Lett 2005:86(12):121113. http://dx doi org/10 1063/11883714.

[64] Petrov GI, Arora R, Yakovlev VV, Wang X, Sokolov AV, Scully MO. Comparison of coherent and spontaneous raman microspectroscopies for noninvasive detection of single bacterial endospores. Proc Natl Acad Sci USA 2007;104(19):7776-9. http://dx.doi.org/10.1073/pnas.0702107104.

[65] Okuno M, Kano H, Leproux P, Couderc V. H. o Hamaguchi, Ultrabroadband multiplex cars microspectroscopy and imaging using a subnanosecond supercontinuum light source in the deep near infrared. Opt Lett 2008;33(9):923-5. http://dx doi org/10 1364/OL 33000923

[66] Selm R, Winterhalder M, Zumbusch A, Krauss G, Hanke T, Sell A, Leitenstorfer A. Ultrabroadband background-free coherent anti-stokes raman scattering microscopy based on a compact er:fiber laser system. Opt Lett 2010;35(19):3282-4. http://dx.doi.org/10.1364/OL.35.003282.

[67] Oron D, Dudovich N, Yelin D, Silberberg Y. Narrow-band coherent anti-stokes raman signals from broad-band pulses. Phys Rev Lett 2002;88:063004.

[68] Frostig H, Katz O, Natan A, Silberberg Y. Single-pulse stimulated raman scattering spectroscopy. Opt Lett 2011;36(7):1248-50. http://dx.doi.org/ 10.1364/OL.36.001248

[69] Katz O, Levitt JM, Grinvald E, Silberberg Y. Single-beam coherent raman spectroscopy and microscopy via spectral notch shaping. Opt Express 2010;18(22):22693-701. http://dx.doi.org/10.1364/OE.18.022693.

[70] Muller M, Schins JM. Imaging the thermodynamic state of lipid membranes with multiplex cars microscopy. J Phys Chem B 2002;106(14):3715-23.

[71] Potma E, Xie X. Detection of single lipid bilayers with coherent anti-stokes raman scattering (cars) microscopy. J Raman Spectrosc 2003:34:642-50.

[72] Larsson K, Rand RP. Detection of changes in environment of hydrocarbon chains by raman-spectroscopy and its application to lipid-protein systems. Biochem Biophys Acta 1973;326:245-55.

[73] Orendorff CJ, Ducey MW, Pemberton JE. Quantitative correlation of raman spectral indicators in determining conformational order in alkyl chains. J Phys Chem A 2002;106:6991-8.

[74] Wurpel G, Schins J, Müller M. Direct measurement of chain order in single phospholipid mono- and bilayers with multiplex cars. J Phys Chem B 2004;108:3400-3.

[75] Wurpel G, Rinia H, Müller M. Imaging orientational order and lipid density in multilamellar vesicles with multiplex cars microscopy. J Microsc 2005;218:37-45.

[76] Potma E, Xie X. Direct visualization of lipid phase segregation in single lipid bilayers with coherent anti-stokes raman scattering microscopy. Chem Phys Chem 2005;6:77-9.

[77] Li L, Cheng JX. Label-free coherent anti-stokes raman scattering imaging of coexisting lipid domains in single bilayers. J Phys Chem B 2008;112(6):1576-9.

[78] Li L, Wang H, Cheng J-X. Quantitative coherent anti-stokes raman scattering imaging of lipid distribution in coexisting domains. Biophys J 2005;89:3480-90.

[79] Konig K, So PTC, Mantulin WW, Gratton E. Cellular response to near-infrared femtosecond laser pulses in two-photon microscopes. Opt Lett 1997;22(2):135-6.

[80] Konig K, Becker TW, Fischer P, Riemann I, Halbhuber KJ. Pulse-length dependence of cellular response to intense near-infrared laser pulses in multiphoton microscopes. Opt Lett 1999;24(2):113-5.

[81] Fu Y, Wang H, Shi R, Cheng J-X. Characterization of photodamage in coherent anti-stokes raman scattering microscopy. Opt Express 2006;14:3942-51.

[82] Brown DA. Lipid droplets: proteins floating on a pool of fat. Curr Biol 2001;11(11):R446-9.

[83] Nan X, Potma E, Xie X. Nonperturbative chemical imaging of organelle transport in living cells with coherent anti-stokes raman scattering microscopy. Biophys J 2006;91:728-35.

[84] Jüngst C, Winterhalder MJ, Zumbusch A. Fast and long term lipid droplet tracking with cars microscopy. J Biophotonics 2011;4(6):435-41.

[85] Lyn RK, Kennedy DC, Stolow A, Ridsdale A, Pezacki JP. Dynamics of lipid droplets induced by the hepatitis c virus core protein. Biochem Biophys Res Commun 2010;399(4):518-24.

[86] Rinia HA, Burger KNJ, Bonn M, Müller M. Quantitative label-free imaging of lipid composition and packing of individual cellular lipid droplets using multiplex cars microscopy. Biophys J 2008;95(10):4908-14.

[87] Bonn M, Müller M, Rinia HA, Burger KNJ. Imaging of chemical and physical state of individual cellular lipid droplets using multiplex cars microscopy. J Raman Spectrosc 2009;40(7):763-9.

[88] Brackmann C, Norbeck J, Akeson M, Bosch D, Larsson C, Gustafsson L, Enejder A. Cars microscopy of lipid stores in yeast: the impact of nutritional state and genetic background. J Raman Spectrosc 2009;40(7):748-56.

[89] Paar M, Jüngst C, Steiner NA, Magnes C, Sinner F, Kolb D, Lass A, Zimmermann R, Zumbusch A, Kohlwein SD, Wolinski H. Remodeling of lipid droplets during lipolysis and growth in adipocytes. J Biol Chem 2012;287(14):11164. 73.

[90] Zhu JB, Lee BG, Buhman KK, Cheng JX. A dynamic, cytoplasmic triacylglycerol pool in enterocytes revealed by ex vivo and in vivo coherent anti-stokes raman scattering imaging. J Lipid Res 2009;50(6):1080-9.

[91] Lee B, Zhu JB, Wolins NE, Cheng JX, Buhman KK. Differential association of adipophilin and tip47 proteins with cytoplasmic lipid droplets in mouse enterocytes during dietary fat absorption. Biochim Biophys Acta Mol Cell Biol Lipids 2009;1791(12):1173-80.

[92] Lee B, Fast AM, Zhu J, Cheng JX, Buhman KK. Intestine-specific expression of acyl coa:diacylglycerol acyltransferase 1 reverses resistance to diet-induced
[93] Su AI, Pezacki JP, Wodicka L, Brideau AD, Supekova L, Thimme R, Wieland S, Bukh J, Purcell RH, Schultz PG, Chisari FV. Genomic analysis of the host response to hepatitis $\mathrm{c}$ virus infection. Proceedings of the national academy of sciences of the USA 2002:99(24):15669-74.

[94] Rakic B, Sagan SM, Noestheden M, Belanger S, Nan XL, Evans CL, Xie XS, Pezacki JP. Peroxisome proliferator-activated receptor alpha antagonism inhibits hepatitis c virus replication. Chem Biol 2006;13(1):23-30.

[95] Lyn RK, Kennedy DC, Sagan SM, Blais DR, Rouleau Y, Pegoraro AF, Xie XS, Stolow A, Pezacki JP. Direct imaging of the disruption of hepatitis c virus replication complexes by inhibitors of lipid metabolism. Virology 2009;394(1):130-42

[96] Le TT, Huff TB, Cheng JX. Coherent anti-stokes raman scattering imaging of lipids in cancer metastasis. Bmc Cancer 9.

[97] Wang HW, Bao N, Le TT, Lu C, Cheng JX. Microfluidic cars cytometry. Opt Express 2008;16(8):5782-9.

[98] Le TT, Cheng JX. Single-cell profiling reveals the origin of phenotypic variability in adipogenesis. PLoS One 2009;4(4):e5189.

[99] Saar BG, Contreras-Rojas LR, Xie XS, Guy RH. Imaging drug delivery to skin with stimulated raman scattering microscopy. Mol. Pharm 2011;8:969-75.

[100] König K, Breunig H, Bückle R, Kellner-Höfer M, Weinigel M, Büttner E, Sterry W, Lademann J. Optical skin biopsies by clinical cars and multiphoton fluorescence/shg tomography. Laser Phys Lett 2011;8:465-8.

[101] Hellerer T, Axäng C, Brackmann C, Hillertz P, Pilon M, Enejder A. Monitoring of lipid storage in caenorhabditis elegans using coherent anti-stokes raman scattering (cars) microscopy. PNAS 2007;104:14658-63.

[102] Le TT, Duren HM, Slipchenko MN, Hu C-D, Cheng J-X. Label-free quantitative analysis of lipid metabolism in living caenorhabditis elegans. J Lipid Res 2010;51:672-7.

[103] Yen K, Le TT, Bansal A, Narasimhan D, Cheng JX, Tissenbaum HA. A comparative study of fat storage quantitation in nematode caenorhabditis elegans using label and label-free methods. Plos One 2010;5(9):650.

[104] Mörcka C, Olsenb L, Kurthc C, Perssona A, Stormb NJ, Svenssona E, Janssond JO, Hellqvista M, Enejderc A, Faergeman NJ, Pilona M. Statins inhibit protein lipidation and induce the unfolded protein response in the non-sterol producing nematode caenorhabditis elegans. PNAS 2009;106:18285-90.

[105] Dou W, Zhang DL, Jung Y, Cheng JX, Umulis DM. Label-free imaging of lipiddroplet intracellular motion in early drosophila embryos using femtosecondstimulated raman loss microscopy. Biophys J 2012:102(7):1666-75.

[106] Bélanger E, Henry FP, Vallée R, Randolph MA, Kochevar IE, Winograd JM, Lin $\mathrm{CP}$, Côté $\mathrm{D}$. In vivo evaluation of demyelination and remyelination in a nerve crush injury model. Biomed Opt Express 2011;2:2698-708.

[107] Lim RS, Kratzer A, Barry NP, Miyazaki-Anzai S, Miyazaki M, Mantulin WW, Levi M, Potma EO, Tromberg BJ. Multimodal cars microscopy determination of the impact of diet on macrophage infi ltration and lipid accumulation on plaque formation in apoe-defi cient mice. J Lipid Res 2010;51:1729-37.

[108] Mansfield J, Moger J, Green E, Moger C, Winlove CP. Chemically specific imaging and in-situ chemical analysis of articular cartilage with stimulated raman scattering. J Biophotonics, in press. http://dx.doi.org/10.1002/ jbio.201200213.

[109] Wang H, Fu Y, Zickmund P, Shi R, Cheng J-X. Coherent anti-stokes raman scattering imaging of axonal myelin in live spinal tissues. Biophys J 2005;89:581-91.

[110] Fu Y, Wang H, Huff TB, Shi R, Cheng J-X. Coherent anti-stokes raman scattering imaging of myelin degradation reveals a calcium-dependent pathway in lyso-ptdcho-induced demyelination. J Neurosci Res 2007;85:2870-81.

[111] Huff T, Cheng J-X. In vivo coherent anti-stokes raman scattering imaging of sciatic nerve tissue. J Microsc 2007;225:175-82.

[112] Shi Y, Kim S, Huff TB, Borgens RB, Park K, Shi R, Cheng JX. Effective repair of traumatically injured spinal cord by nanoscale block copolymer micelles. Nat Nanotechnol 2010;5:80-7.

[113] Huff TB, Shi Y, Sun W, Wu W, Shi R, Cheng J-X. Real-time cars imaging reveals a calpain-dependent pathway for paranodal myelin retraction during highfrequency stimulation. PLoS ONE 2011;6:e17176.

[114] Galli R, Uckermann O, Winterhalder MJ, Sitoci-Ficici KH, Geiger KD, Koch E, Schackert G, Zumbusch A, Steiner G, Kirsch M. Vibrational spectroscopic imaging and multiphoton microscopy of spinal cord injury. Anal Chem 2012;84(20):8707-14.

[115] Evans CL, Xu X, Kesari S, Xie XS, Wong ST, Young GS. Chemically-selective imaging of brain structures with cars microscopy. Opt Express 2007:15:12076-87.

[116] Pohling C, Buckup T, Pagenstecher A, Motzkus M. Chemoselective imaging of mouse brain tissue via multiplex cars microscopy. Biomed Opt Express 2011;2:2110-6.

[117] Meyer T, Bergner N, Bielecki C, Krafft C, Akimov D, Romeike BFM, Reichart R, Kalff R, Dietzek B, Popp J. Nonlinear microscopy, infrared, and Raman microspectroscopy for brain tumor analysis. J Biomed Optics 2011;16:021113.

[118] Moger J, Garrett NL, Begley D, Mihoreanu L, Lalatsa A, Lozano MV, Mazza M, Schatzleinc A, Uchegbuc I. Imaging cortical vasculature with stimulated raman scattering and two-photon photothermal lensing microscopy. J Raman Spectrosc 2012;43:668-74.

[119] Kim S-H, Lee E-S, Lee JY, Lee ES, Lee B-S, Park JE, Moon DW. Multiplex coherent anti-stokes raman spectroscopy images intact atheromatous lesions 
and concomitantly identifies distinct chemical profiles of atherosclerotic lipids. Circ Res 2010;106:1332-41.

[120] Le TT, Langohr IM, Locker MJ, Sturek M, Cheng J-X. Label-free molecula imaging of atherosclerotic lesions using multimodal nonlinear optical microscopy. J Biomed Optics 2007;12:054007.

121] Wang H-W, Le TT, Cheng J-X. Label-free imaging of arterial cells and extracellular matrix using a multimodal cars microscope. Opt Commun 2008;281:1813-22.

[122] Lim RS, Suhalim JL, Miyazaki-Anzai S, Miyazaki M, Levi M, Potma EO, Tromberg BJ. Identification of cholesterol crystals in plaques of atherosclerotic mice using hyperspectral cars imaging. J Lipid Res 2011:52:2177-86.

[123] Ko ACT, Ridsdale A, Smith MSD, Mostaço-Guidolin LB, Hewko MD, Pegoraro AF, Kohlenberg EK, Schattka B, Shiomi M, Stolow A, Sowaa MG. Multimoda nonlinear optical imaging of atherosclerotic plaque development in myocardial infarction-prone rabbits. J Biomed Opt 2010;15:020501.

[124] Wang H-W, Mattew VS, Locker J, Cheng J-X, Stureka M. Stent-induced coronary artery stenosis characterized by multimodal nonlinear optica microscopy. J Biomed Opt 2011;16:021110.

[125] Wu Y-M, Chen H-C, Chang W-T, Jhan J-W, Lin H-L, Liau I. Quantitative assessment of hepatic fat of intact liver tissues with coherent anti-stokes raman scattering microscopy. Anal Chem 2009;81:1496-504.

[126] Lin J, Lu F, Zheng W, Xu S, Tai D, Yu H, Huang Z. Assessment of liver steatosis and fibrosis in rats using integrated coherent anti-stokes raman scattering and multiphoton imaging technique. J Biomed Opt 2011;16:116024.

[127] Gao L, Li F, Thrall MJ, Yang Y, Xing J, Hammoudi AA, Zhao H, Massoud Y, Cagle PT, Fan Y, Wong KK, Wang Z, Wong STC. On-the-spot lung cancer differentia diagnosis by label-free, molecular vibrational imaging and knowledge-based classification. J Biomed Opt 2011;16:096004.
[128] Yang Y, Li F, Gao L, Wang Z, Thrall MJ, Shen SS, Wong KK, Wong STC Differential diagnosis of breast cancer using quantitative, label-free and molecular vibrational imaging. Biomed Opt Express 2011;2:2160-74.

[129] Yue SH, Cardenas-Mora JM, Chaboub LS, Lelievre SA, Cheng JX. Label-free analysis of breast tissue polarity by raman imaging of lipid phase. Biophys J 2012;102(5):1215-23.

[130] Salameh T, Le T, Nichols M, Bauer E, Cheng J, Camarillo I. An ex vivo coculture model system to evaluate stromal-epithelial interactions in breast cancer. Int J Cancer 2013;132:288-96.

[131] Brackmann C, Zaborowska M, Sundberg J, Gatenholm P, Enejder A. In situ imaging of collagen synthesis by osteoprogenitor cells in microporous bacterial cellulose scaffolds. Tissue Eng Part C 2012;18:227-34.

[132] Benitez PL, Sweet JA, Fink H, Chennazhi KP, Nair SV, Enejder A, Heilshorn SC. Sequence-specific crosslinking of electrospun, elastin-like protein preserves bioactivity and native-like mechanics. Adv Healthcare Mater.

[133] Brackmann C, Dahlberg J-O, Vrana NE, Lally C, Gatenholm P, Enejder A. Nonlinear microscopy of smooth muscle cells in artificial extracellular matrices made of cellulose. J Biophotonics 2012;5:404-14.

[134] Moger J, Johnston BD, Tyler CR. Imaging metal oxide nanoparticles in biological structures with cars microscopy. Opt Express 2008:16(5): 3408-19.

[135] Mazza M, Notman R, Anwar J, Rodger A, Hicks M, Parkinson G, McCarthy D, Daviter T, Moger J, Garrett N, Mead T, Briggs M, Schätzlein AG, Uchegbu IF. Nanofiber-based delivery of therapeutic peptides to the brain. ACS Nano, in press. http://dx.doi.org/10.1021/nn305193d.

[136] Garrett NL, Lalatsa A, Uchegbu I, Schätzlein A, Moger J. Exploring uptake mechanisms of oral nanomedicines using multimodal nonlinear optical microscopy. J Biophotonics 2012;5:458-68. 